\title{
Epitaxial growth, magnetic properties, and lattice dynamics of Fe nanoclusters on GaAs(001)
}

\author{
B. Roldan Cuenya* and A. Naitabdi \\ Physics Department, University of Central Florida, Orlando, Florida 32816-2385, USA
}

E. Schuster, R. Peters, M. Doi ${ }^{\dagger}$ and W. Keune

Fachbereich Physik, Universität Duisburg-Essen (Campus Duisburg), D-47048 Duisburg, Germany

(Received 13 September 2005; revised manuscript received 3 May 2007; published 10 September 2007)

\begin{abstract}
Epitaxial bcc-Fe(001) ultrathin films have been grown at $\sim 50{ }^{\circ} \mathrm{C}$ on reconstructed $\mathrm{GaAs}(001)-(4 \times 6)$ surfaces and investigated in situ in ultrahigh vacuum (UHV) by reflection high-energy electron diffraction, scanning tunneling microscopy (STM), x-ray photoelectron spectroscopy (XPS), and ${ }^{57} \mathrm{Fe}$ conversion electron Mössbauer spectroscopy (CEMS). For $t_{\mathrm{Fe}}=1 \mathrm{ML}$ (monolayer) Fe coverage, isolated Fe nanoclusters are arranged in rows along the [110] direction. With increasing $t_{\mathrm{Fe}}$ the Fe clusters first connect along the [-110], but not along the [110] direction at $2.5 \mathrm{ML}$, then consist of percolated Fe clusters without a preferential orientation at $3 \mathrm{ML}$, and finally form a nearly smooth film at $4 \mathrm{ML}$ coverage. Segregation of Ga atoms within the film and on the Fe surface appears to occur at $t_{\mathrm{Fe}}=4 \mathrm{ML}$, as evidenced by XPS. For coverages below the magnetic percolation, temperature-dependent in situ CEMS measurements in zero external field provided superparamagnetic blocking temperatures $T_{B}$ of $62 \pm 5,80 \pm 10$, and $165 \pm 5 \mathrm{~K}$ for $t_{\mathrm{Fe}}=1.9,2.2$, and $2.5 \mathrm{ML}$, respectively. At $T<T_{B}$, freezing of superparamagnetic clusters is inferred from the observed quasilinear $T$ dependence of the mean hyperfine magnetic field $\left\langle B_{h f}\right\rangle$. By combining the STM and CEMS results, we have determined a large magnetic anisotropy constant of $\sim 5 \times 10^{5}$ and $\sim 8 \times 10^{5} \mathrm{~J} / \mathrm{m}^{3}$ at $t_{\mathrm{Fe}}=1.9-2.2$ and $2.5 \mathrm{ML}$, respectively. For $t_{\mathrm{Fe}} \leqslant 2.5 \mathrm{ML}$, our uncoated "free" Fe clusters exhibit intrinsic magnetic ordering below $T_{B}$, contrary to literature reports on metal-coated Fe clusters on GaAs. Our present results demonstrate that the nature of the percolation transition for free $\mathrm{Fe}$ nanoclusters on $\mathrm{GaAs}(001)$ in $\mathrm{UHV}$ is from superparamagnetism to ferromagnetism. From the Mössbauer spectral area, a very low Debye temperature $\Theta_{D}$ of $196 \pm 4 \mathrm{~K}$ is deduced for these uncoated Fe nanoclusters in UHV, indicating a strong phonon softening in the clusters.
\end{abstract}

DOI: 10.1103/PhysRevB.76.094403 PACS number(s): 76.80.+y, 75.75.+a, 61.46. $-\mathrm{w}, 75.20 .-\mathrm{g}$

\section{INTRODUCTION}

The structural, magnetic, and electronic properties of Fe films deposited on GaAs have been studied extensively over the last decades and continue to attract strong interest. ${ }^{1}$ $\mathrm{Fe} / \mathrm{GaAs}$ is an interesting candidate among ferromagnet/ semiconductor (SC) heterostructures for the realization of next-generation spintronic devices. ${ }^{2-4}$ In fact, the Fe/GaAs-based hybrid system has been shown to be a promising device since it allows the manipulation of the SC spin polarization via electron spin injection from the FM. ${ }^{5-12}$

GaAs is an ideal substrate for the epitaxial growth of $\mathrm{Fe}(001)$ in the stable bcc phase since the lattice parameter of $\mathrm{Fe}$ is approximately half that of the zinc-blende-type GaAs $\left(2 a_{\mathrm{Fe}} / a_{\mathrm{GaAs}}=1.014\right.$, corresponding to a small lattice misfit of $1.4 \%$ ). Nominally, this implies a small in-plane compressive stress for the Fe film. The achievement of single-crystal epitaxy is important since for many applications, one needs to exploit the magnetic anisotropy of the material. Therefore, amorphous, polycrystalline, or even oriented polycrystalline films are not acceptable.

High quality epitaxial growth of $\mathrm{Fe}$ on $\mathrm{GaAs}(001)$ and GaAs(110) substrates by molecular beam epitaxy (MBE) has been widely reported in the literature, ${ }^{13-55}$ together with detailed investigations of the magnetic properties, ${ }^{15-18,20-30,32-38,40-53}$ e.g., the evolution of the ferromagnetic order $22-25,32,40,45,48,51$ or the relationship between uniaxial and cubic magnetic anisotropies as a function of layer thickness. ${ }^{18,22,33,34,42-44,47,48,50,53}$ Some of these properties have been related to the interfacial compound formation and the atomic scale nucleation processes for various Gaand As-terminated $\mathrm{GaAs}(100)$ substrate surface reconstructions. ${ }^{19,21,23}$ Schönherr et al. ${ }^{39}$ studied the growth of Fe on the (100), (311)A, and (331)A GaAs surfaces and its dependence on the growth temperature and termination of the GaAs surface, in an attempt to obtain macroscopically smooth Fe layers. They found that As-rich surfaces favored the formation of smooth $\mathrm{Fe}$ layers, if grown at low temperatures. Gordon et al. ${ }^{31}$ showed that $\mathrm{Fe}$ films grown on an S-terminated surface exhibit a nearly cubic structure. By contrast, a tetragonal distortion was observed when deposited on the GaAs- $(4 \times 6)$ surface.

The successful growth of smooth epitaxial Fe layers with minimum interdiffusion at the $\mathrm{Fe} / \mathrm{GaAs}$ interface is of relevance for the successful injection of highly spin-polarized electrons. The use of As-rich GaAs reconstructions has been shown to reduce the density of defects in the Fe layers. ${ }^{39}$ It is also well known, however, that nonferromagnetic Fe-As compounds may form at the As-rich Fe/GaAs interface, producing interfacial "magnetic dead layers," ${ }^{26}$ or $\mathrm{Fe}_{3} \mathrm{Ga}_{2-x} \mathrm{As}_{x}$ interlayers with only about half of the magnetization of bulk bcc-Fe (Refs. 28 and 29) due to the interdiffusion of As into the Fe overlayer. From such an interface, the injection of highly spin-polarized electrons into the semiconductor will become unlikely since a high spin polarization and large ferromagnetically ordered moments, even in the first metallic monolayers at the interface, are a necessary condition. Considerable efforts were devoted to gain insight into the effect of $\mathrm{Ga}$ and As interdiffusion across the Fe overlayer on the 
magnetic properties and to explain the origin of the nonmagnetic layer occurring at the $\mathrm{Fe} / \mathrm{GaAs}$ interface upon hightemperature Fe deposition. ${ }^{36}$ Such interdiffusion was observed by several experimental techniques, including Auger electron spectroscopy and x-ray photoelectron spectroscopy. ${ }^{15,20,30,35,36,43,51,56,57}$ The dissociation of Ga and As atoms from the surface of GaAs, followed by their outdiffusion across thin $\mathrm{Fe}$ layers, results in the formation of Fe-based alloys which critically alter the magnetic phase at the interface. ${ }^{28-30}$ Meanwhile, several methods were explored to reduce the segregation of As onto the Fe surface. It is now established that the growth of the Fe film at or near room temperature (RT), ${ }^{23,24,26,27,35}$ or at very low temperatures, ${ }^{37}$ reduces the interdiffusion and interface roughness. Another possible way of reducing As interdiffusion and segregation of As to the surface is to grow the Fe films on Ga-rich surfaces. ${ }^{23,26,27,35,41,52}$ In our previous works, ${ }^{35,41,52}$ it was demonstrated by means of Mössbauer spectroscopy that it is possible to preserve a high Fe magnetic moment at the interface of epitaxial $\mathrm{Fe} / \mathrm{GaAs}(001)-(4 \times 6), \mathrm{Fe} / \mathrm{GaAs}(001) / \mathrm{Al}_{0.35} \mathrm{Ga}_{0.65} \mathrm{As}(001)$, and $\mathrm{Fe} / \mathrm{GaAs}(001)$-based light-emitting diodes. Further, a bulklike $\mathrm{Fe}$ spin moment at the $\mathrm{Fe} / \mathrm{GaAs}(100)-(4 \times 6)$ interface was directly observed by $\mathrm{x}$-ray magnetic circular dichroism. ${ }^{46}$ By means of an appropriate selection of the growth parameters, the formation of nonmagnetic interfacial compounds can be avoided.

This paper focuses on the structural and magnetic properties of small $\mathrm{Fe}$ clusters epitaxially grown on $\mathrm{GaAs}(001)-(4 \times 6)$. Although the epitaxial $\mathrm{Fe} / \mathrm{GaAs}(001)$ system has been extensively studied, there is still a debate over the magnetic properties of the first few monolayers (MLs) and, in particular, over the critical Fe thickness for the onset of ferromagnetism. For example, ferromagnetism (FM) in $\mathrm{Fe}$ films grown at $175^{\circ} \mathrm{C}$ on As-terminated $\mathrm{GaAs}(001)-(2 \times 4)$ surfaces was reported to appear at RT at a critical coverage of $6 \mathrm{ML} \mathrm{Fe}^{22}$ For Fe films grown at RT on Ga-terminated $\operatorname{GaAs}(001)-(4 \times 6)$ surfaces long-rangeordered FM at RT was observed by Xu et al. ${ }^{23}$ at and above a thickness of about $5 \mathrm{ML} \mathrm{Fe}$. Since in the initial state $\mathrm{Fe}$ on $\mathrm{GaAs}(001)$ is known to grow at RT in the Volmer-Weber mode [i.e., by nucleation of isolated three-dimensional (3D) islands], the Fe film is discontinuous until a certain coverage is reached, where island coalescence occurs. Based on magneto-optic Kerr effect (MOKE) measurements, Xu et $a l .{ }^{23}$ suggested that $\mathrm{Fe}$ growth at RT proceeds from a "nonmagnetic" Fe phase for the first 3.5 ML Fe to a short-rangeordered FM (superparamagnetic) phase up to about $5 \mathrm{ML} \mathrm{Fe}$ coverage, followed by FM above $5 \mathrm{ML}$ Fe. Freeland et al. ${ }^{25}$ obtained a critical value of 3.8 ML from MOKE susceptibility measurements, while Bensch et al. ${ }^{32}$ reported a similar critical value of 3.6 ML Fe coverage, obtained from MOKE ac-susceptibility measurements. A crucial question is whether a local ferromagnetic order exists before the onset of the long-range-ordered FM phase. Bensch et al. ${ }^{32}$ claimed that during the initial stages of Fe growth on $\mathrm{GaAs}(001)$, islands are formed that are smaller than the superparamagnetic limit until they coalesce. In principle, the onset of FM may be triggered by a superparamagnetism to FM transition or by a phase transition from nonferromagnetic (i.e., paramagnetic) state to FM. ${ }^{32}$ Thus, the magnetic response before the onset of FM could originate either from paramagnetism (as Bensch et al. proposed in Ref. 32) or from superparamagnetism of $\mathrm{Fe}$ islands (as suggested by Xu et al., ${ }^{23}$ Freeland et $a l .,{ }^{25}$ and Steinmüller et $a l .{ }^{45}$ ). The absence of magnetization at RT for coverages below $\approx 3.5 \mathrm{ML}$ may, in principle, be caused by interfacial intermixing of Fe with As and Ga (dilute Fe-alloy formation), reducing the Curie temperature $T_{c}$ to below RT), the formation of interfacial nonferromagnetic compounds, or superparamagnetism. Also, the coating material $(\mathrm{Au}, \mathrm{Al}$, or $\mathrm{Cr})$ used by many researchers to protect their Fe films from oxidation during ex situ studies might have an influence on the magnetism of ultrathin Fe layers via interfacial intermixing. Therefore, the investigation of uncoated ("free") $\mathrm{Fe} / \mathrm{GaAs}(001)$ ultrathin films in UHV is very important. Although a number of reports on in situ ultrahigh vacuum (UHV) room-temperature studies on uncoated $\mathrm{Fe} / \mathrm{GaAs}(001)$ films exist, ${ }^{18,21-25,38,45,51}$ in situ investigations performed at low temperatures (near or at magnetic saturation) are scarce..$^{53}$

In the present work, we have investigated the magnetic properties of uncoated (free) Fe clusters on Ga-terminated $\mathrm{GaAs}(001)-(4 \times 6)$ surfaces at $1.9,2.2$, and $2.5 \mathrm{ML}$ Fe coverages by in situ conversion electron Mössbauer spectroscopy (CEMS) in UHV in the temperature range between $27 \mathrm{~K}$ and RT. These coverages are below the critical Fe coverage for ferromagnetism. Moreover, in situ scanning tunneling microscopy (STM), x-ray photoelectron spectroscopy (XPS), and reflection high-energy electron diffraction (RHEED) measurements provided information on cluster growth, atomic interdiffusion, and film structure, respectively. The present results allow us to clarify the nature of the percolation transition observed for uncoated ultrathin epitaxial Fe films on $\mathrm{GaAs}(001)$ in UHV.

\section{EXPERIMENT}

For the preparation and in situ investigation of our samples, we have used two independent UHV systems for MBE called UHV-1 and UHV-2. Details on the epitaxial growth of ultrathin $\mathrm{Fe}(001)$ films on $\mathrm{GaAs}(001)-(4 \times 6)$ are given in our previous articles. ${ }^{35,41,52}$

STM and XPS measurements were carried out in system UHV-1 at Orlando, a UHV system equipped with three interconnected chambers that allows in situ sample preparation and multitechnique characterization. The substrates used in these experiments are pieces of an undoped "epiready" GaAs(001) wafer (purchased from Wafer Technology Ltd). The substrates were cleaned by acetone, followed by 2-propanol before being transferred into the MBE system (base pressure in the low $10^{-10}$ mbar range). The substrates were then annealed in $\mathrm{UHV}$ at $590{ }^{\circ} \mathrm{C}$ for $20 \mathrm{~min}$ to remove adsorbed impurities and the native oxide from the surface. Subsequently, the temperature was increased to $600{ }^{\circ} \mathrm{C}$ and maintained while the first cycle of $\mathrm{Ar}^{+}$ion sputtering was performed at an energy of $0.5 \mathrm{keV}$ for $90 \mathrm{~min}$. This sputtering cycle was followed by annealing at $600{ }^{\circ} \mathrm{C}$ for $30 \mathrm{~min}$ for optimum surface healing. This treatment was repeated 
twice, whereupon the complete removal of oxygen and carbon was observed by XPS. Under similar preparation conditions, but on doped GaAs(001), Moosbühler et al. ${ }^{33}$ and Ionescu et $a l .{ }^{49}$ observed by STM large terraces (more than $50 \mathrm{~nm}$ long and $50 \mathrm{~nm}$ wide) with ordered Ga-terminated $(4 \times 2)$ and $(2 \times 6)$ reconstructions. A mixture of $(4 \times 2)$ and $(2 \times 6)$ reconstructions has also been observed by STM on RT-sputtered and subsequently annealed $\operatorname{GaAs}(001)$ surfaces. ${ }^{58,59}$ High-purity Fe was deposited nominally at RT and at a pressure better than $6 \times 10^{-10} \mathrm{mbar}$ using an e-beam evaporator with a constant rate of $0.05 \AA / \mathrm{s}$, as monitored by a calibrated quartz crystal microbalance. Finally, the sample was transferred in situ to the STM chamber (pressure in the high $10^{-11}$ mbar range), where STM measurements were performed at RT using an etched W tip previously cleaned by $\mathrm{Ar}^{+}$ion sputtering. The STM images were acquired at constant current mode with the voltage applied to the sample.

RHEED, low-energy electron diffraction (LEED), Auger electron spectroscopy (AES), and ${ }^{57} \mathrm{Fe}$ CEMS were performed in situ in UHV in the MBE chamber of the UHV-2 system at Duisburg (base pressure $\leqslant 1.4 \times 10^{-10} \mathrm{mbar}$ ). The $\mathrm{GaAs}(001)$ substrates used in these experiments had identical characteristics to the ones described above and were cleaned in an analogous way. After this treatment, no surface impurities were detected by AES, and a RHEED image typical of the "pseudo- $(4 \times 6)$ " reconstruction of the Ga-terminated GaAs(001) surface was observed (see below). Immediately after surface cleaning, the Mössbauer-active ${ }^{57} \mathrm{Fe}$ isotope (95.5\% isotopical enrichment, 99.95 at. \% purity) was deposited nominally at RT from a Knudsen cell $\left(\mathrm{Al}_{2} \mathrm{O}_{3}\right.$ crucible) at a pressure $p \leqslant 1.5 \times 10^{-9}$ mbar. However, the real substrate temperature during growth, $T_{s}$, was $\sim 40-50{ }^{\circ} \mathrm{C}$, as measured by a thermocouple fixed to the sample holder. The deposition rate $(0.02-0.03 \AA / \mathrm{s})$ and film thickness were monitored with a quartz crystal microbalance, which had been calibrated shortly before the preparation by RHEED intensity oscillations during the epitaxial growth of a fcc$\mathrm{Fe}(001)$ thin film on a clean $\mathrm{Cu}(001)$ surface at RT. ${ }^{60}$ Also, RHEED intensity oscillations observed above a coverage of $\sim 5 \mathrm{ML}$ of the growing $\mathrm{Fe}(001)$ film on $\mathrm{GaAs}(001)-(4 \times 6)$ have been used for thickness calibration. The precision of this thickness determination is estimated to be $10 \%$. During Fe growth RHEED images were continuously monitored by a charge coupled device camera and occasionally supported by LEED images. After preparation and structural characterization by RHEED and/or LEED, the sample was in situ transferred and attached to the cold finger of a UHVcompatible liquid-helium cryostat for ${ }^{57} \mathrm{Fe}$ CEMS in the temperature range between $27 \mathrm{~K}$ and $\mathrm{RT}$ in a zero-external field. A ${ }^{57} \mathrm{Co}$ source (Rh matrix) of $\sim 100 \mathrm{mCi}$ activity was mounted on the Mössbauer drive outside the UHV system. The $\gamma$ radiation passed through a UHV-tight, high-purity Be window and hit the sample at perpendicular incidence relative to the film plane. A channeltron was used to detect the conversion electrons emitted from the sample surface during the Mössbauer resonance.

After the CEMS measurements in UHV, the ${ }^{57} \mathrm{Fe}$ films were coated by a protective Pt cap layer of $40 \AA$ (grown at a rate of $0.04 \AA / s$ at RT and evaporated from an electron gun).
Subsequently, the Pt-coated samples were investigated $e x$ situ by CEMS at RT. Throughout our paper, the temperature of $300 \mathrm{~K}$ represents room temperature. A proportional counter filled with high-purity $\mathrm{He}-4 \% \mathrm{CH}_{4}$ gas with the sample mounted inside was employed for ex situ CEMS at RT. The $\gamma$-ray direction was perpendicular to the sample surface. The proportional counter could be placed between the poles of an electromagnet in order to acquire CEM spectra at RT in an external magnetic field oriented within the sample plane. The CEM spectra were fitted using a least-squares method by the program NORMOS by Brand. ${ }^{61}$

\section{RESULTS AND DISCUSSION}

\section{A. Growth and structural properties}

\section{Low-energy electron diffraction and reflection high-energy electron diffraction}

Details on the epitaxial growth of ultrathin $\mathrm{Fe}(001)$ films on $\operatorname{GaAs}(001)-(4 \times 6)$ are given in our previous articles. ${ }^{35,41,52}$ In our previous RHEED studies, we observed the disappearance of the $\mathrm{GaAs}(001)-(4 \times 6)$-surface reconstruction after deposition of $5.5 \mathrm{ML}$ of $\mathrm{Fe}$, and the appearance of a $(1 \times 1)$ pattern was observed up to a coverage of 42 ML. Our present investigations support and extend our earlier observations. Typical LEED and RHEED patterns of the clean $\operatorname{GaAs}(001)-(4 \times 6)$ substrate are shown in Figs. 1(a)-1(d). These diffraction patters are characteristic of the pseudo- $(4 \times 6)$ reconstruction [with mixed $(4 \times 2)$ and $(2 \times 6)$ reconstructions] of the clean Ga-terminated $\operatorname{GaAs}(001)$ surface. A schematic of the LEED pattern is shown in Fig. 1(b), where the fundamental (superstructure) reflections are indicated by large (small) circles. Our present diffraction results are in agreement with the work by Zölfl $e t$ $a l .{ }^{26}$ and Madami et al. ${ }^{47}$ and with our previous work. ${ }^{35,41,52}$

RHEED patterns taken at different $\mathrm{Fe}$ coverages are shown in Figs. 1(d) and 1(f)-1(j). After the initial Fe deposition [Figs. 1(d) and 1(f)-1(h)], i.e., at Fe coverages up to 3.6 ML, the GaAs superstructure reflections disappear, but the fundamental reflections are still observable, although they fade with increasing coverage until they disappear at 3.6-4 ML Fe coverages. This demonstrates that an incomplete island-like Fe film is formed, consisting of Fe nanoclusters. No reflections of bcc-Fe are seen up to $\sim 3.1 \mathrm{MLFe}$. This could indicate that thinner Fe films grow in a structurally disordered (amorphous) state, as mentioned by Bensch et al. ${ }^{34}$ At $3.1 \mathrm{ML}$ coverage, weak indications of bcc- $\mathrm{Fe}(001)$ reflections appear, superimposed on weak fundamental $\mathrm{GaAs}(001)$ diffraction spots. The $\mathrm{GaAs}(001)$ reflections disappear at $\sim 3.6-4.0 \mathrm{ML} \mathrm{Fe}$, while the bcc-Fe streaks increase in intensity [Fig. 1(i)]. The type of diffraction pattern observed for $\mathrm{Fe}$ in the range of $\sim 3.6-4.0 \mathrm{ML}$ indicates epitaxial, 3D Fe(001) island growth. ${ }^{35}$ Above 4 ML Fe coverage, the Fe reflections gain in intensity, indicating the formation of a closed Fe film covering the entire substrate. Thus, coalescence of Fe clusters occurs at $\sim 3.6-4.0 \mathrm{ML}$, and after 5 ML no significant change in the RHEED pattern was observed. This is demonstrated in the RHEED pattern of 93 ML Fe(001) [Fig. 1(j)], which is the typical 3D diffraction 


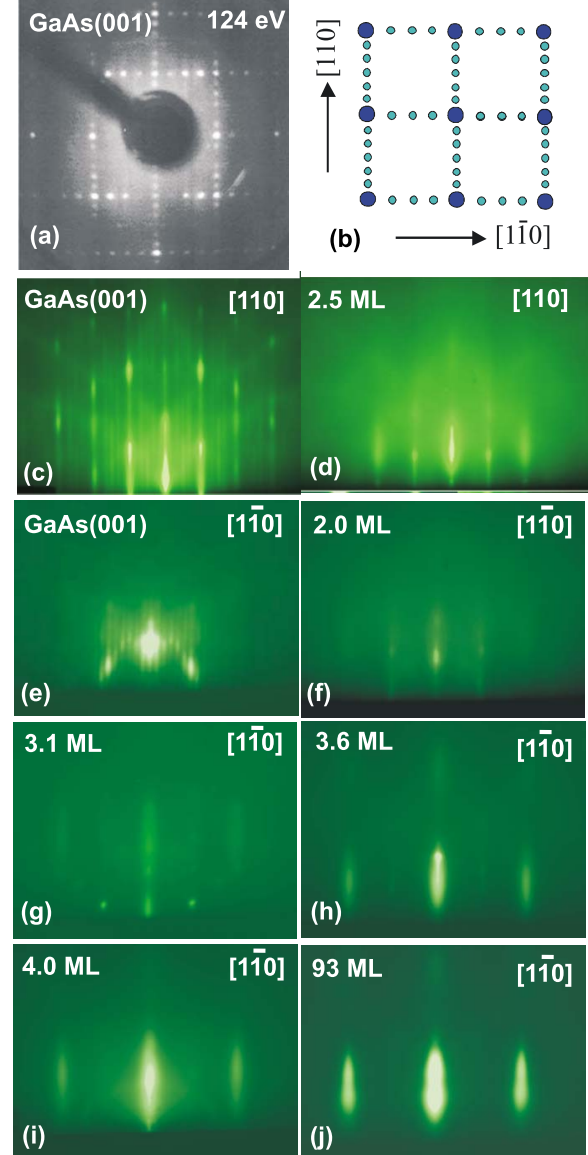

FIG. 1. (Color online) (a) LEED pattern of the clean $\mathrm{GaAs}(001)-(4 \times 6)$ surface measured with $124 \mathrm{eV}$ electron energy. (b) Schematics of the LEED diffraction spots of the GaAs(001)- $(4 \times 6)$ surface reconstruction. Images (c) and (d) display RHEED patterns measured with the electron beam along the [110] azimuthal direction of the clean and 2.2 ML Fe covered GaAs(001) surface, respectively. RHEED patterns measured with the beam (e) along $[1-10]$ of clean $\mathrm{GaAs}(001)-(4 \times 6)$ and at $\mathrm{Fe}$ coverages of (f) $2 \mathrm{ML}$, (g) $3.1 \mathrm{ML}$, (h) $3.6 \mathrm{ML}$, (i) $4.0 \mathrm{ML}$, and (j) 93 ML (electron energy: $15 \mathrm{keV}$ ).

pattern of a surface being rough on an atomic scale. Above 5 ML coverage, RHEED intensity oscillations with 1 ML $\mathrm{Fe}(001)$ periodicity were observed with increasing coverage. The appearance of these oscillations suggests that a smoothing of the $\mathrm{Fe}$ surface and quasi-layer-by-layer type of $\mathrm{Fe}$ growth occurs on the smoothed surface formed after the coalescence of the $\mathrm{Fe}$ islands. These results (for growth at $40^{\circ}-$ $50^{\circ} \mathrm{C}$ ) are in agreement with previous reports by Brockmann et al. ${ }^{27}$ on RT grown $\mathrm{Fe} / \mathrm{GaAs}(001)$ samples. They found by STM that the roughness amplitude of 1-2 ML in height remained unchanged with increasing Fe thickness, indicating a quasi-layer-by-layer growth mode which leaves the surface structure of the Fe films unchanged. For growth at $150{ }^{\circ} \mathrm{C}$, however, Gester et al. ${ }^{18}$ found pyramidlike structures and a step density which increased approximately linearly with film thickness.
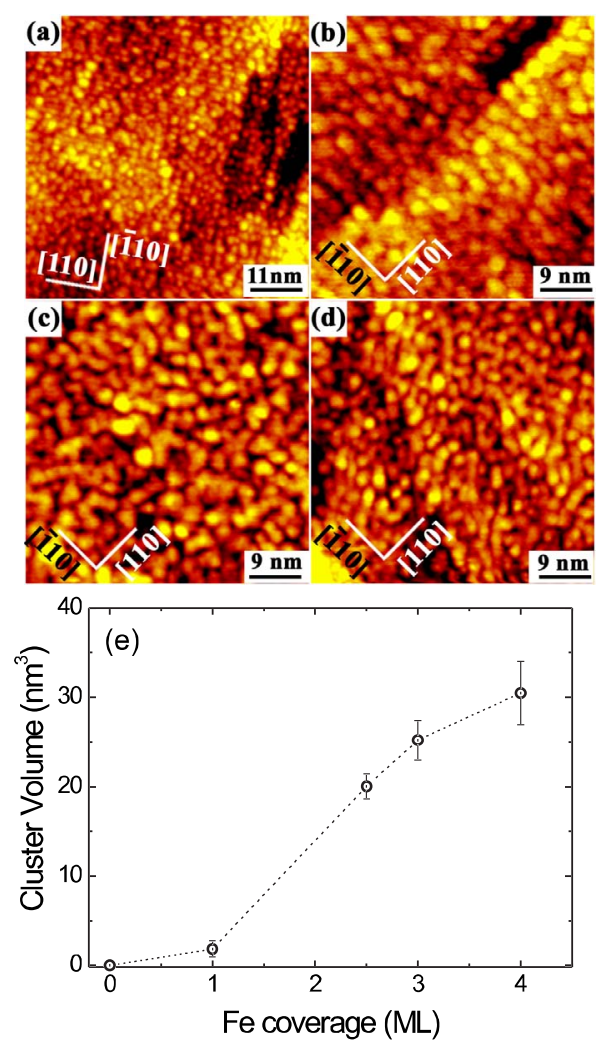

FIG. 2. (Color online) STM images of four different coverages of Fe on GaAs(001): (a) $1 \mathrm{ML}$, (b) $2.5 \mathrm{ML}$, (c) $3 \mathrm{ML}$, and (d) 4 ML. The tunneling parameters were (a) $V_{t}=0.5 \mathrm{~V}, I_{t}=0.11 \mathrm{nA}$, (b) $V_{t}$ $=0.3 \mathrm{~V}, I_{t}=0.4 \mathrm{nA}$, (c) $V_{t}=0.5 \mathrm{~V}, I_{t}=0.3 \mathrm{nA}$, and (d) $V_{t}=0.32 \mathrm{~V}$, $I_{t}=0.31 \mathrm{nA}$. Fe clusters arranged parallel to the [-110] rows are observed for the (b) $2 \mathrm{ML} \mathrm{Fe} \mathrm{sample,} \mathrm{while} \mathrm{enhanced} \mathrm{cluster} \mathrm{per-}$ colation is observed when the Fe coverage is increased to (c) $3 \mathrm{ML}$ and (d) 4 ML. (e) Average cluster volume obtained by STM as a function of $\mathrm{Fe}$ coverage.

\section{Scanning tunneling microscopy and $x$-ray photoelectron spectroscopy}

The structural growth of $\mathrm{Fe}$ on the $\mathrm{GaAs}(001)$ pseudo $(4 \times 6)$ surface was followed by in situ STM measurements. This surface exhibits domains of two different Ga-rich reconstructions [the Ga-rich $(4 \times 2)$ and the less Ga-rich $(2 \times 6)$ due to As termination $\left.{ }^{33,49,62-64}\right]$. Figure 2 shows four STM images acquired on $\mathrm{Fe} / \mathrm{GaAs}(001)$ surfaces and corresponding to thicknesses ranging from 1 to 4 ML. Figure 2(a) displays the arrangement of Fe after the deposition of $1 \mathrm{ML}$. Small Fe clusters ordered along parallel rows and separated by a distance of $1.6 \pm 0.2 \mathrm{~nm}$ can be observed among some random domains. This parallel arrangement is related to the nucleation of Fe clusters on top of As rows along the [110] direction that are characteristic of the GaAs(001)- $(4 \times 2)$ reconstruction, [Fig. 2(a), upper left part]. The typical distance between two As dimer rows in the $(4 \times 2)$ reconstruction corresponds to $1.6 \mathrm{~nm} \cdot{ }^{33,49,62-64}$ At this coverage, round $\mathrm{Fe}$ clusters are observed. This growth is consistent with the presence of a Ga-rich surface where the diffusion of $\mathrm{Fe}$ monomers is energetically unfavorable. ${ }^{49}$ At this coverage, a 
single atomic step of the Fe domain, with a height of $\sim 1.5 \pm 0.2 \AA$ corresponding to one monolayer of bcc-Fe, can be observed. However, Fe clusters with $2 \mathrm{ML}$ height $(\sim 2.9 \AA)$ appear as well. This indicates the tendency of Fe to build $\mathrm{Fe}-\mathrm{Fe}$ bonds instead of diffusing on top of $\mathrm{Ga}$ atoms. The growth of $\mathrm{Fe}$ is strongly influenced by the underlying reconstruction, leading to the nucleation of almost circular clusters with a height of 1-2 ML and arranged in rows along the [110] direction, in agreement with previous studies. ${ }^{33,49}$

The increase of the Fe coverage to $2.5 \mathrm{ML}$ [Fig. 2(b)] leads to a more homogeneous size distribution of Fe clusters. Unlike the structure obtained in the case of $1 \mathrm{ML}$ coverage, where the distribution is dominated by 1 ML high clusters $(\sim 1.5 \AA)$, the Fe clusters in the $2.5 \mathrm{ML}$ thick sample are mostly 2 ML high $(\sim 2.9 \AA)$ and present larger diameters $(\sim 29.5 \pm 3.0 \AA)$. This distribution suggests that the Fe clusters grow in lateral size once the $2 \mathrm{ML}$ height has been reached. Besides, Fe clusters arrange into parallel lines separated by a distance of $\sim 25.3 \AA$, which is close to the typical spacing $(24 \AA)$ between parallel As rows along the [-110] direction in the $\operatorname{GaAs}(001)-(2 \times 6)$ reconstruction. ${ }^{33,49,62-64}$ This arrangement is reminiscent of an initial GaAs(001)- $(2 \times 6)$ reconstruction where As rows represent the favorable nucleation sites for Fe atoms. In the early growth stages, the initial deposition of $\mathrm{Fe}$ atoms on As sites and in its vicinities (missing Ga dimers) is expected. Further increase in the coverage leads to a saturation in the diameter of the Fe clusters $(\sim 29 \AA)$ along the [110] direction, while additional nucleation appears in the [-110] direction. As can be observed in the STM image of Fig. 2(b), Fe clusters appear connected along the $[-110]$ direction as a result of the additional nucleation occurring when the diameter limit is attained. It is noteworthy that at this coverage (2.5 ML) no connectivity between Fe clusters is noticed along the [110] direction, suggesting that the onset of ferromagnetism at RT would not arise at this coverage.

Upon increasing the deposition to $3 \mathrm{ML}$, the surface morphology of this coverage consists of percolated $\mathrm{Fe}$ islands resulting from diffusion and coalescence of smaller Fe clusters [Fig. 2(c)]. At this thickness, the Fe domains are randomly distributed and the corresponding percolations exhibit no preferential orientation. Thus, the influence of the reconstruction is no longer observed at this coverage, suggesting a layer-by-layer growth with a surface roughness of $2 \mathrm{ML}$ height $(\sim 2.9 \AA)$. The growth of Fe from 2.5 to $3 \mathrm{ML}$ takes place by the bridging of $\mathrm{Fe}$ atoms across $\mathrm{Ga}$ rows along the [110] direction when the initial reconstruction is $(2 \times 6)$ and along the $[-110]$ orientation in the case of the $(4 \times 2)$ surface. The structural percolation of Fe domains is associated with the onset of the ferromagnetic phase, which is reported to occur at room temperature for a critical Fe thickness in the range of $3 \pm 0.5 \mathrm{ML}$ to $4.8 \mathrm{ML}$ (Refs. $23-25,32,40,45,48$, and 51) on $\mathrm{GaAs}(001)$ surfaces. Increasing the coverage to 4 ML leads to a relatively smoothed surface compared to the previous 3 ML morphology, but with the same surface roughness of 2 ML height [Fig. 2(d)]. Similarly, Zölfl et al. ${ }^{26}$ and Brockmann et al. ${ }^{27}$ reported coalescence of Fe islands for a coverage between 3 and 4 ML followed by quasi-layerby-layer growth after $5 \mathrm{ML} \mathrm{Fe}$ deposition on sputter-

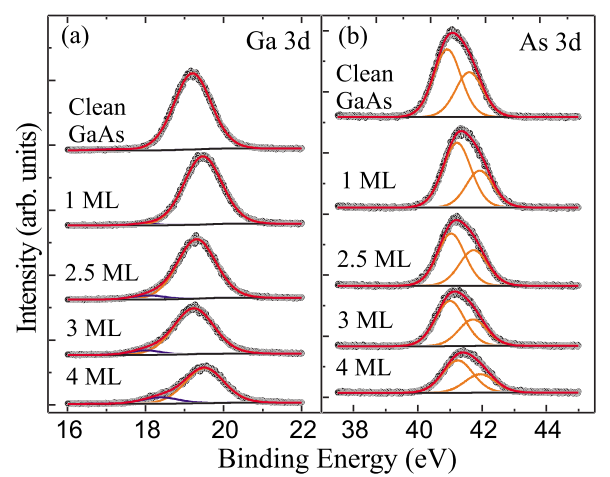

FIG. 3. (Color online) Ga $3 d$ and As $3 d$ core level photoelectron spectra as a function of Fe coverage. The spectra labeled "clean GaAs" were measured after the cleaning treatment described in the experimental section, leading to the $\operatorname{GaAs}(001)-(4 \times 6)$ surface.

annealed Ga-rich GaAs(001) surfaces. Our STM results are in agreement with the conclusions drawn from our RHEED results described above.

The effect of intermixing between the Fe overlayer and $\mathrm{Ga}$ and As atoms of the substrate was investigated by XPS measurements. Figure 3 shows peak intensities of the Ga $3 d$ and As $3 d$ core levels acquired after cleaning by sputtering and annealing the substrate and after the deposition of 1-4 $\mathrm{ML}$ of Fe. The binding energies (BEs) of $3 d$ core levels of $\mathrm{Ga}$ and As $\left(3 d_{5 / 2}, 3 d_{3 / 2}\right)$ measured before Fe deposition are $19.2 \mathrm{eV}$ and $(40.9 \mathrm{eV}, 41.6 \mathrm{eV})$, respectively. Those values are in agreement with what has been previously obtained for a clean GaAs surface. ${ }^{65}$ The deposition of $1 \mathrm{ML}$ of $\mathrm{Fe}$ on this freshly prepared surface induced a moderate increase in the $\mathrm{BE}$ of both $\mathrm{Ga}$ and As $3 d$ levels. This shift to higher BE with comparable values for $\mathrm{Ga} 3 d_{5 / 2}(\sim 0.26 \mathrm{eV})$ and As $3 d_{5 / 2}$ $(\sim 0.30 \mathrm{eV})$ indicates a substantial change in their atomic coordination at the $\mathrm{Fe} / \mathrm{GaAs}$ interface. The increase in the binding energy can be interpreted as a result of a downward band bending effect taking place at the $\operatorname{GaAs}(001)$ surface, which creates extrinsic surface states and a negatively charged $\mathrm{GaAs}(001)-\mathrm{Fe}$ interface. This initial shift of the core level to a higher binding energy is commonly observed when a clean semiconductor surface is exposed to small amounts of metallic atoms ( $\sim 1$ ML coverage) due to charge transfer effects. ${ }^{66}$

Due to the low density of $\mathrm{Fe}$ clusters and the lack of $\mathrm{Fe}$ mobility at this coverage, the $\mathrm{Fe}-\mathrm{Fe}$ coordination must be insignificant, resulting in predominantly $\mathrm{Fe}-\mathrm{GaAs}$ interactions as the origin of the moderate shift in the $\mathrm{BE}$ of $\mathrm{Ga}$ and As $3 d$ levels. As the thickness of Fe is increased to $2.5 \mathrm{ML}$, only slight shifts are observed in the $\mathrm{BE}$ of $\mathrm{Ga} 3 d$ $(\sim 0.10 \mathrm{eV})$ and As $3 d_{5 / 2}(0.15 \mathrm{eV})$ levels. This effect can be attributed to the increase of $\mathrm{Fe}-\mathrm{Fe}$ coordination, which is energetically more favorable than $\mathrm{Fe}-\mathrm{Ga}$ bonds, resulting in a significant reduction of the Fe-substrate interactions. The tendency of the $3 d \mathrm{BE}$ of $\mathrm{Ga}$ and As atoms at the interface to return to their original values before $\mathrm{Fe}$ deposition indicates the lack of stable $\mathrm{Fe}-\mathrm{Ga}$ and $\mathrm{Fe}-\mathrm{As}$ bonds. The absence of intermixing between $\mathrm{As}$ and $\mathrm{Fe}$ is evident if we consider the evolution of the XPS $3 d$ levels in Fig. 3(b), where no shoul- 
der at higher BE can be observed in the As $3 d$ core level spectra characteristic of $\mathrm{Fe}-\mathrm{As}$. Such a shoulder has been observed for $\mathrm{Fe}$ on the As-rich $\mathrm{GaAs}(001)-c(8 \times 2)$ surface and was attributed to interstitial As in the Fe overlayer. ${ }^{56} \mathrm{We}$ did not observe this effect here. Moreover, the $\mathrm{BE}$ of As $3 d_{5 / 2}$ for coverages higher than $2.5 \mathrm{ML}$ exhibits a remarkable stability. Thus, we infer that no diffusion of As into the Fe layer and no As segregation on the Fe surface occurs below $4 \mathrm{ML} \mathrm{Fe}$. Reduced intermixing (relative to Asterminated surfaces) was previously reported for $\mathrm{Ga}-$ terminated surfaces where As is depleted from the surface prior to Fe deposition. ${ }^{36}$ However, the diffusion of Ga atoms through the Fe overlayer becomes evident by the appearance of an additional peak in the XPS spectra at lower BE $(18.0 \mathrm{eV})$ [Fig. 3(a)]. The measured BE corresponds to the value of a pure $\mathrm{Ga}\left(3 d_{5 / 2}\right)$ level, indicating the presence of low-coordinated $\mathrm{Ga}$ atoms at the surface. The intensity of this shoulder in the XPS spectra was found to increase with increasing $\mathrm{Fe}$ thickness and can be attributed to $\mathrm{Ga}$ atoms diffusing to the surface of the Fe film. The diffusion of Ga atoms through $\mathrm{Fe}$ overlayers has been previously demonstrated. ${ }^{51,56}$ However, segregation at the Fe surface is generally prevented by the presence of As. This effect can be understood in terms of energy minimization since based on the heats of formation for the $\mathrm{Fe}_{2} \mathrm{As}$ and $\mathrm{Fe}_{2} \mathrm{Ga}$ phases $\left(\Delta H_{\mathrm{Fe}_{2} \mathrm{As}}=-38 \mathrm{~kJ} / \mathrm{mol}\right.$ and $\left.\Delta H_{\mathrm{Fe}_{2} \mathrm{Ga}}=-16 \mathrm{~kJ} / \mathrm{mol}\right),{ }^{36}$ the $\mathrm{Fe}-\mathrm{As}$ bond is more favorable than $\mathrm{Fe}-\mathrm{Ga}$. In our study, the segregation of $\mathrm{Ga}$ atoms at the $\mathrm{Fe}$ surface can take place since we did not observe any As diffusion that may inhibit such segregation process. This behavior was also theoretically predicted by Mirbt et al. ${ }^{67}$ The attenuation of $\mathrm{Ga}$ and As intensities as a function of the Fe coverage gives further insight into this diffusion effect [Fig. 4(a)]. The attenuation of Ga and As $3 d$ intensities exhibits slightly different trends, with the Ga signal decreasing more slowly than the As one. This effect can be attributed to the presence of dissolved $\mathrm{Ga}$ atoms in the Fe overlayers and on the surface of the topmost Fe layer, as predicted theoretically by Erwin et al. ${ }^{68}$ and observed above 3 ML coverage by Giovanelli et al. ${ }^{51}$

Figure 4(b) (inset) shows the XPS spectra collected for the Fe $2 p$ core level as a function of the coverage from 1 to 4 ML. For all Fe thicknesses, a similar binding energy of $\sim 706.8 \mathrm{eV}\left(\mathrm{Fe} 2 p_{3 / 2}\right.$ level) is measured. This value is in agreement with what is expected for bulk Fe, indicating the stability of the chemical environment of $\mathrm{Fe}$, even for the lowest $\mathrm{Fe}$ coverages investigated here and confirming the absence of strong $\mathrm{Fe}-\mathrm{Ga}$ and $\mathrm{Fe}-\mathrm{As}$ bonds. Since the magnetic moment in Fe is carried by $3 d$ orbitals and those orbitals are usually involved in the interactions with other chemical species, the reduced intermixing detected in our samples indicates that those orbitals should have bulklike characteristics. Therefore, the onset of ferromagnetism is expected to occur at lower $\mathrm{Fe}$ thicknesses than if significant intermixing was involved. Then, this onset should mainly depend on the coalescence of the Fe islands, with a minimum influence of $\mathrm{Fe}-\mathrm{Ga}$ and Fe-As intermixing. Our STM results reveal the beginning of $\mathrm{Fe}$ coalescence at $\sim 2.5 \mathrm{ML}$ (Fig. 2), with nearly complete cluster coarsening into domains when the $\mathrm{Fe}$ thickness is about 3 ML. The XPS results are in agreement

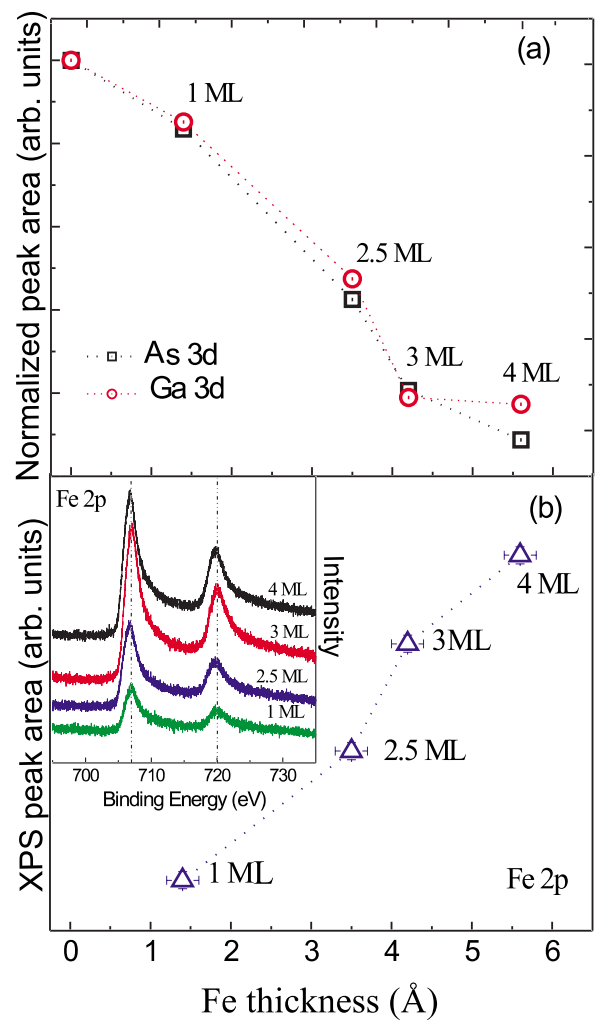

FIG. 4. (Color online) (a) Normalized integrated XPS signals from Ga $3 d$ (circles) and As $3 d$ (squares) measured in situ (UHV) as a function of $\mathrm{Fe}$ coverage on clean $\mathrm{GaAs}(001)$. The areas of the $\mathrm{Ga}$ and As peaks in the $\operatorname{GaAs}(001)$ substrate have been used for the normalization. (b) Integrated XPS signal of the Fe $2 p$ core level (triangles) and raw Fe $2 p$ XPS spectra (insert) measured as a function of Fe coverage on clean $\operatorname{GaAs}(001)$. (The dotted lines are a guide for the eye.)

with the morphology data since a clear increase of the Fe $2 p$ intensities is observed from 1 to $3 \mathrm{ML}$ coverages [Fig. 4(b)]. For an Fe thickness of $\sim 3$ ML, a change in the slope can be observed in the increase of the $\mathrm{Fe} 2 p$ intensities, which is attributed to the effect of the coalescence of the Fe clusters: The contribution of $\mathrm{Fe}$ atoms from the surrounding small islands is screened by the topmost layer as the Fe clusters start coalescing. Additionally, the segregation of $\mathrm{Ga}$ atoms to the surface of the topmost Fe layer will reduce the $\mathrm{Fe} 2 p$ intensity.

\section{Mössbauer spectroscopy}

Figure 5(a) displays CEM spectra measured at RT in situ in UHV (UHV-2) on ${ }^{57} \mathrm{Fe}(001) / \mathrm{GaAs}(001)$ samples with 2.5, 2.2, and $1.9 \mathrm{ML}{ }^{57} \mathrm{Fe}$ coverages (from top to bottom, respectively). The spectra shown in Fig. 5(b) were taken $e x$ situ at RT after coating the same Fe films with a $40 \AA$ thick protective Pt film. All spectra in Fig. 5 were measured in zero-external field $\left(B_{\text {ext }}=0 \mathrm{~T}\right)$, except the bottom spectrum in Fig. 5(b), which was obtained ex situ in an external field of $B_{\text {ext }}=1.3 \mathrm{~T}$ applied in the film plane of the Pt-coated 1.9 ML ${ }^{57} \mathrm{Fe}$ sample. All spectra could be least-squares fitted with a slightly asymmetric quadrupole-split doublet, except the bot- 


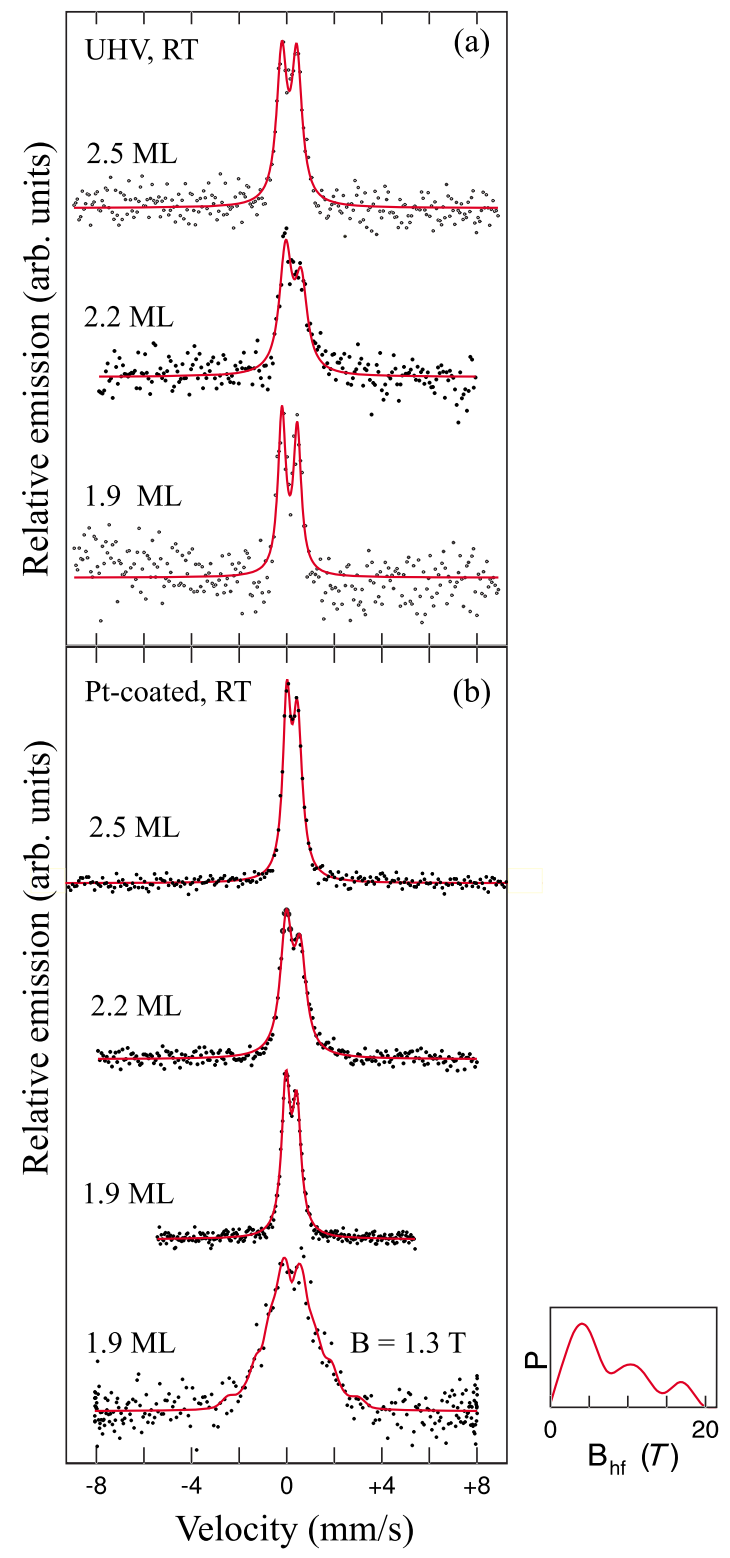

FIG. 5. (Color online) CEM spectra taken at room temperature on ${ }^{57} \mathrm{Fe}(001) / \mathrm{GaAs}(001)-(4 \times 6)$ for ${ }^{57} \mathrm{Fe}$ coverages of $2.5,2.2$, and 1.9 ML. (a) in situ in UHV (free ${ }^{57} \mathrm{Fe}$ surface) and (b) ex situ (Pt covered ${ }^{57} \mathrm{Fe}$ surface). Bottom spectrum in (b): $1.9 \mathrm{ML}{ }^{57} \mathrm{Fe}$ coverage measured ex situ in $B_{\text {ext }}=1.3 \mathrm{~T}$. (All other spectra were measured at $B_{e x t}=0 \mathrm{~T}$.) The full-drawn line is a least-squares fit with a quadrupole doublet except in the bottom spectrum of (b), which is fitted according to a distribution of hyperfine fields $P\left(B_{h f}\right)$ (right-hand side).

tom spectrum in Fig. 5(b). The Mössbauer parameters obtained from the fitting are given in Table I. The values of the isomer shift $\delta$ of all samples are found to be positive with respect to $\delta=0.0 \mathrm{~mm} / \mathrm{s}$ of pure bulk bcc-Fe (our reference absorber), indicating a decrease of the $s$-electron density at the ${ }^{57} \mathrm{Fe}$ nucleus at the ${ }^{57} \mathrm{Fe} / \mathrm{GaAs}(001)$ interface. This decrease of the $s$-electron density may arise from electronic hybridization of wave functions of $\mathrm{Fe}$ and neighboring As and/or Ga atoms, as well as from the expansion of the average $\mathrm{Fe}-\mathrm{Fe}$ distance in the film (relative to that of bulk bcc-Fe at RT) ${ }^{69}$ On average, the Pt coating resulted in an increase of the $\delta$ values by $+0.04 \pm 0.05 \mathrm{~mm} / \mathrm{s}$ relative to those of the uncoated Fe films. This indicates a further reduction of the $s$-electron density at the ${ }^{57} \mathrm{Fe}$ nucleus due to Pt.

The observed quadrupole splitting $\Delta E_{Q}$ of the doublet is relatively large for the uncoated $\mathrm{Fe}$ films $\left(\Delta E_{Q}\right.$ $\approx 0.64 \mathrm{~mm} / \mathrm{s}$ on the average, Table I) but is remarkably reduced (to an average value of $\Delta E_{Q} \approx 0.50 \mathrm{~mm} / \mathrm{s}$ ) after $\mathrm{Pt}$ coating. The pure observation of an electric quadrupole splitting in Fig. 5 is a qualitative proof of the noncubic local environment around the ${ }^{57} \mathrm{Fe}$ atom in these ${ }^{57} \mathrm{Fe}$ nanoclusters on $\operatorname{GaAs}(001)$, whether free or Pt coated. One could think that the reason for this symmetry breaking is the small lattice mismatch of $1.4 \%$ between bcc-Fe and GaAs that would lead to a small in-plane lattice compression (and, consequently, to a lattice expansion along the film-normal direction) in the $\mathrm{Fe}$ nanoclusters. However, contrary to expectation, the in-plane atomic spacing in epitaxial $\mathrm{Fe}(001)$ films grown at $40-50{ }^{\circ} \mathrm{C}$ on $\mathrm{GaAs}(001)$ has been demonstrated by RHEED to increase up to $2.5 \%$ (relative to that of bulklike bcc-Fe films) for Fe coverages below $5 \AA,{ }^{35}$ most likely due to some atomic intermixing at the $\mathrm{Fe} / \mathrm{GaAs}$ interface. This in-plane expansion could result in a lattice contraction along the filmnormal direction. Our XPS results (Sec. III A 2) suggest the presence of Ga impurities rather than As impurities on the surface of the Fe clusters. Since Ga atoms are larger than Fe atoms, the incorporation of $\mathrm{Ga}$ atoms in bcc-Fe at substitutional lattice sites can explain the observed increase of the atomic spacing in the nanoclusters. Positive isomer shifts (as given in Table I) are typically observed for $\mathrm{Fe}_{1-x} \mathrm{Ga}_{x}$ solid solutions. $^{70}$

The slight asymmetry observed in the intensity of the two lines in the quadrupole doublets [Figs. 5(a) and 5(b)] proves that a preferred direction of the main component $V_{z z}$ of the electric field gradient (EFG) exists in the epitaxial ${ }^{57} \mathrm{Fe}$ nanoclusters on GaAs(001). According to Table I, the average line intensity ratio $A_{21}$ is equal to 0.83 and $0.86 \mathrm{~mm} / \mathrm{s}$ [averaged over all Pt-coated and uncoated (UHV) nanoclusters, respectively]. For simplicity, we assume a uniaxial EFG with $V_{z z}$ $\neq 0$ and a zero asymmetry parameter $(\eta=0)$. Then, the average angle $\langle\varphi\rangle$ between the direction of $V_{z z}$ and the $\gamma$-ray direction (or film-normal direction) can be determined from the ratio $A_{21}$ of the integrated intensity of line 2 (with more positive velocity) and line 1 (with more negative velocity) according to the relation: ${ }^{71}$

$$
A_{21}=\frac{\frac{2}{3}+\sin ^{2} \varphi}{1+\cos ^{2} \varphi} \quad \text { or } \quad A_{21}=\frac{1+\cos ^{2} \varphi}{\frac{2}{3}+\sin ^{2} \varphi}
$$

for $V_{z z}<0$ or $V_{z z}>0$, respectively. By using the average values of $A_{21}=0.86$ and 0.83 for all uncoated (UHV) and all Pt-coated samples, respectively, we obtain from Eq. (1) values of $\langle\varphi\rangle=61^{\circ}$ and $63^{\circ}$ for ${ }^{57} \mathrm{Fe} / \mathrm{GaAs}(001)$ and $\mathrm{Pt} /{ }^{57} \mathrm{Fe} / \mathrm{GaAs}(001)$, respectively, assuming $V_{z z}>0$ (average angle of $62^{\circ}$ ). If we assume $V_{z z}<0$, the $\langle\varphi\rangle$ values are $49^{\circ}$ and $47^{\circ}$ for ${ }^{57} \mathrm{Fe} / \mathrm{GaAs}(001)$ and $\mathrm{Pt} /{ }^{57} \mathrm{Fe} / \mathrm{GaAs}(001)$, re- 
TABLE I. ${ }^{57} \mathrm{Fe}$ Mössbauer parameters measured at room temperature: isomer shift $\delta$ (relative to bulk bcc-Fe at RT), quadrupole splitting $\Delta E_{Q}$, Lorentzian linewidth $\Gamma$ (full width at half maximum) of the Lorentzian line, ratio of the spectral areas of lines 2 and $1, A_{21}$, quadrupole nuclear line shift $2 \varepsilon$, average hyperfine field $\left\langle B_{h f}\right\rangle$, and root mean square (rms) of $P\left(B_{h f}\right)$ distribution. The data are extracted from Fig. 5.

\begin{tabular}{|c|c|c|c|c|}
\hline$T=\mathrm{RT}$ & $\begin{array}{c}\text { Isomer } \\
\text { shift } \\
\delta(\mathrm{mm} / \mathrm{s})\end{array}$ & $\begin{array}{l}\text { Quadrupole } \\
\text { splitting } \\
\Delta E_{Q}(\mathrm{~mm} / \mathrm{s})\end{array}$ & $\begin{array}{l}\text { Lorentzian } \\
\text { linewidth } \\
\Gamma(\mathrm{mm} / \mathrm{s})\end{array}$ & $\begin{array}{c}\text { Line intensity } \\
\text { ratio } \\
A_{21} \\
\end{array}$ \\
\hline \multicolumn{5}{|c|}{${ }^{57} \mathrm{Fe}(2.5 \mathrm{ML}) / \mathrm{GaAs}(001)$} \\
\hline $\begin{array}{l}\text { As deposited } \\
B_{e x t}=0 \mathrm{~T} \text {, in situ }\end{array}$ & $0.25 \pm 0.01$ & $0.63 \pm 0.02$ & $0.52 \pm 0.03$ & $0.98 \pm 0.07$ \\
\hline $\begin{array}{l}\mathrm{Pt}(40 \AA) \text { coated } \\
B_{\text {ext }}=0 \mathrm{~T}, \text { ex situ }\end{array}$ & $0.312 \pm 0.004$ & $0.447 \pm 0.006$ & $0.44 \pm 0.01$ & $0.89 \pm 0.03$ \\
\hline \multicolumn{5}{|c|}{${ }^{57} \mathrm{Fe}(2.2 \mathrm{ML}) / \mathrm{GaAs}(001)$} \\
\hline $\begin{array}{l}\text { As deposited } \\
B_{\text {ext }}=0 \mathrm{~T} \text {, in situ }\end{array}$ & $0.36 \pm 0.01$ & $0.65 \pm 0.02$ & $0.62 \pm 0.03$ & $0.72 \pm 0.07$ \\
\hline $\begin{array}{l}\mathrm{Pt}(40 \AA) \text { coated } \\
B_{\text {ext }}=0 \mathrm{~T}, \text { ex situ }\end{array}$ & $0.35 \pm 0.01$ & $0.59 \pm 0.01$ & $0.59 \pm 0.02$ & $0.76 \pm 0.04$ \\
\hline \multicolumn{5}{|c|}{${ }^{57} \mathrm{Fe}(1.9 \mathrm{ML}) / \mathrm{GaAs}(001)$} \\
\hline $\begin{array}{l}\text { As deposited } \\
B_{\text {ext }}=0 \mathrm{~T} \text {, in situ }\end{array}$ & $0.25 \pm 0.01$ & $0.65 \pm 0.02$ & $0.40 \pm 0.03$ & $0.89 \pm 0.08$ \\
\hline $\begin{array}{l}\mathrm{Pt}(40 \AA) \text { coated } \\
B_{\text {ext }}=0 \mathrm{~T}, \text { ex situ }\end{array}$ & $0.32 \pm 0.01$ & $0.46 \pm 0.01$ & $0.43 \pm 0.01$ & $0.83 \pm 0.02$ \\
\hline $\begin{array}{l}\mathrm{Pt}(40 \AA) \text { coated } \\
B_{\text {ext }}=1.3 \mathrm{~T} \text {, ex situ }\end{array}$ & $0.35 \pm 0.04$ & $\begin{array}{c}2 \varepsilon= \\
-0.022 \pm 0.08\end{array}$ & $\begin{array}{c}\text { average }\left\langle B_{h f}\right\rangle \\
7.6 \pm 0.3 \mathrm{~T}\end{array}$ & $\begin{array}{l}\mathrm{rms} B_{h f} \\
6.3 \mathrm{~T}\end{array}$ \\
\hline
\end{tabular}

spectively (average angle of $48^{\circ}$ ). $V_{z z}$ is canted relative to the film-normal direction by these angles. Unfortunately, the sign of $V_{z z}$ is unknown; it can be determined, in principle, by applying a strong magnetic field.

Irrespective of the unknown sign of $V_{z z}$, the $\langle\varphi\rangle$ values obtained provide evidence for a significant in-plane component of $V_{z z}$. Two reasons may be responsible for this phenomenon: (i) a preferred noncubic atomic environment of $\mathrm{Ga}$ atoms (less likely As atoms, as our XPS results show) around the ${ }^{57} \mathrm{Fe}$ atom in the intermixed interfacial region (as opposed to a random interfacial alloy) and/or (ii) an anisotropic in-plane lattice distortion superimposed to the tetragonal distortion along the film-normal direction. Such an in-plane lattice strain has been made responsible for the observed strong uniaxial magnetic anisotropy in the thin epitaxial Fe films on $\operatorname{GaAs}(001)$ in a recent theory, ${ }^{67}$ and was observed experimentally. ${ }^{55}$ Future angular dependent CEMS studies at grazing incidence of the $\gamma$ ray could provide information about the preferred direction of the in-plane component $V_{z z}$.

\section{B. Magnetic properties}

\section{Room temperature}

An open question is whether the ${ }^{57} \mathrm{Fe}$ nanoclusters on $\mathrm{GaAs}(001)$ at RT are either in a paramagnetic state, or in a superparamagnetic state above the blocking temperature $T_{B}$ (or, alternatively, in a state of thermally fluctuating spin clusters above a critical magnetic ordering temperature $T_{C}$ ).
While the paramagnetic state will respond only very weakly to an external field $B_{\text {ext }}$, the superparamagnetic state (above $T_{B}$ ) or the state of correlated spin fluctuations (above $T_{C}$ ), may show a strong response to $B_{\text {ext }}$. The external field will block the rapid thermal superparamagnetic fluctuations (or the thermal fluctuations of spin clusters above $T_{C}$ ) and cause the appearance of a Zeeman sextet with a relatively large hyperfine field splitting. ${ }^{72,73}$ On the other hand, the Mössbauer spectrum in the case of paramagnetism will be only weakly affected by $B_{\text {ext }}$.

Figure 5(b) (bottom spectrum) shows the result of an ex situ CEMS measurement at RT with the Pt-coated 1.9 ML ${ }^{57} \mathrm{Fe} / \mathrm{GaAs}(001)$ sample placed in a field $B_{\text {ext }}$ of $1.3 \mathrm{~T}$. The drastic broadening observed for this in-field spectrum, as compared to the zero-field spectrum for the same 1.9 ML ${ }^{57} \mathrm{Fe}$ sample, unambiguously demonstrates the presence of a relatively large effective hyperfine magnetic field induced by the external field via slowing down of superparamagnetic fluctuations or critical fluctuations of spin clusters. The Mössbauer parameters obtained from spectral least-squares fitting with a distribution of hyperfine magnetic fields $P\left(B_{h f}\right)$ [Fig. 5(c), bottom spectrum, right-hand side] are shown in Table I (bottom row). The distribution of hyperfine fields can be the result of both the cluster size distribution and the alloy formation near the Fe/GaAs interface. The most striking feature is the relatively large average (effective) hyperfine field $\left\langle B_{h f}\right\rangle$ of $7.6 \mathrm{~T}$ induced by the much smaller external field of $1.3 \mathrm{~T}$. This proves that the ${ }^{57} \mathrm{Fe}$ nanoclusters in the $1.9 \mathrm{ML}$ ${ }^{57} \mathrm{Fe} / \mathrm{GaAs}(001)$ sample (which have the smallest average 
cluster size in all our samples) are either superparamagnetic at RT or show critical spin-cluster fluctuations at RT $>T_{C}$. Paramagnetism can be definitively ruled out at RT because in that case the measured (effective) hyperfine field would be about equal to the external field. We may conclude that also the ${ }^{57} \mathrm{Fe}$ clusters in the 2.2 and $2.5 \mathrm{ML}{ }^{57} \mathrm{Fe} / \mathrm{GaAs}(001)$ samples (containing larger clusters) are superparamagnetic (or show critical spin-cluster fluctuations) at RT. Similar CEM spectra on the latter samples, taken at RT and in an external field (not shown), exhibit analogous broadening effects as the one shown in Fig. 5(b) (bottom spectrum).

The magnitude of the measured (effective) average hyperfine field $\left\langle B_{h f}\right\rangle$ is given by $\left\langle B_{h f}\right\rangle=\left\langle B_{\text {int }}\right\rangle-B_{\text {ext }}$, where $\left\langle B_{\text {int }}\right\rangle$ is the magnitude of the average intrinsic hyperfine field (assuming collinearity of all fields as in a ferromagnet). Using $B_{e x t}=1.3 \mathrm{~T}$ and $\left\langle B_{h f}\right\rangle=7.6 \mathrm{~T}$, we obtain a value of $\left\langle B_{\text {int }}\right\rangle$ $=8.9 \mathrm{~T}$ for the nanoclusters at RT. This value should be compared with $\left\langle B_{\text {int }}\right\rangle=33.0 \mathrm{~T}$ for bulk bcc-Fe at RT. The main factor responsible for the smaller $\left\langle B_{\text {int }}\right\rangle$ value observed in the nanoclusters is the incomplete blocking of the superparamagnetic or thermal spin-cluster fluctuations by the relatively weak external field; i.e., a complete static spin alignment along $B_{\text {ext }}$ is not yet achieved. Other factors include alloying effects and nanocluster size effects.

Although it is possible to exclude paramagnetism at RT from a Mössbauer measurement in an external field, such a measurement cannot distinguish between superparamagnetic fluctuations $\left(T>T_{B}\right)$ and critical fluctuations of spin clusters $\left(T>T_{C}\right)$. However, we will show below that the measured $T$ dependence of the average hyperfine magnetic field $\left\langle B_{h f}\right\rangle$ provides strong evidence of superparamagnetism and not of critical fluctuations of spin clusters near a phase transition.

\section{Low temperature}

Figures 6-8 display CEM spectra from uncoated ${ }^{57} \mathrm{Fe} / \mathrm{GaAs}(001)$ samples at $1.9,2.2$, and $2.5 \mathrm{ML}{ }^{57} \mathrm{Fe}$ coverages, respectively. The spectra were measured in situ in UHV-2, in zero-external field, as a function of temperature from $27 \mathrm{~K}$ to RT. At RT, the CEM spectra consist of the central quadrupole-split doublet due to superparamagnetism, as described above. With decreasing temperature, the overall width of the spectra first increases only slightly until below a certain temperature a drastic broadening occurs due to a sudden onset of magnetic hyperfine splitting. This behavior indicates a transition from superparamagnetism to ferromagnetism of the Fe clusters in all three samples upon cooling, as will be outlined in more detail below. Since the thermal fluctuations of the net magnetization in a superparamagnetic cluster are faster than the Mössbauer observation time (being equal to the Larmor precession time of $\sim 10^{-8} \mathrm{~s}$ of the ${ }^{57} \mathrm{Fe}$ nuclear magnetic moment), the time-averaged hyperfine field measured in the superparamagnetic state (i.e., for temperatures above the blocking temperature $T_{B}$ ) by the Mössbauer effect in the absence of an external magnetic field becomes zero, and the effect is insensitive to the internal (local) ferromagnetic order within the Fe clusters. In Figs. 6-8, at the lowest temperature, i.e., in the ferromagnetic state, magnetic hyperfine-split spectra are observed for all samples due to the

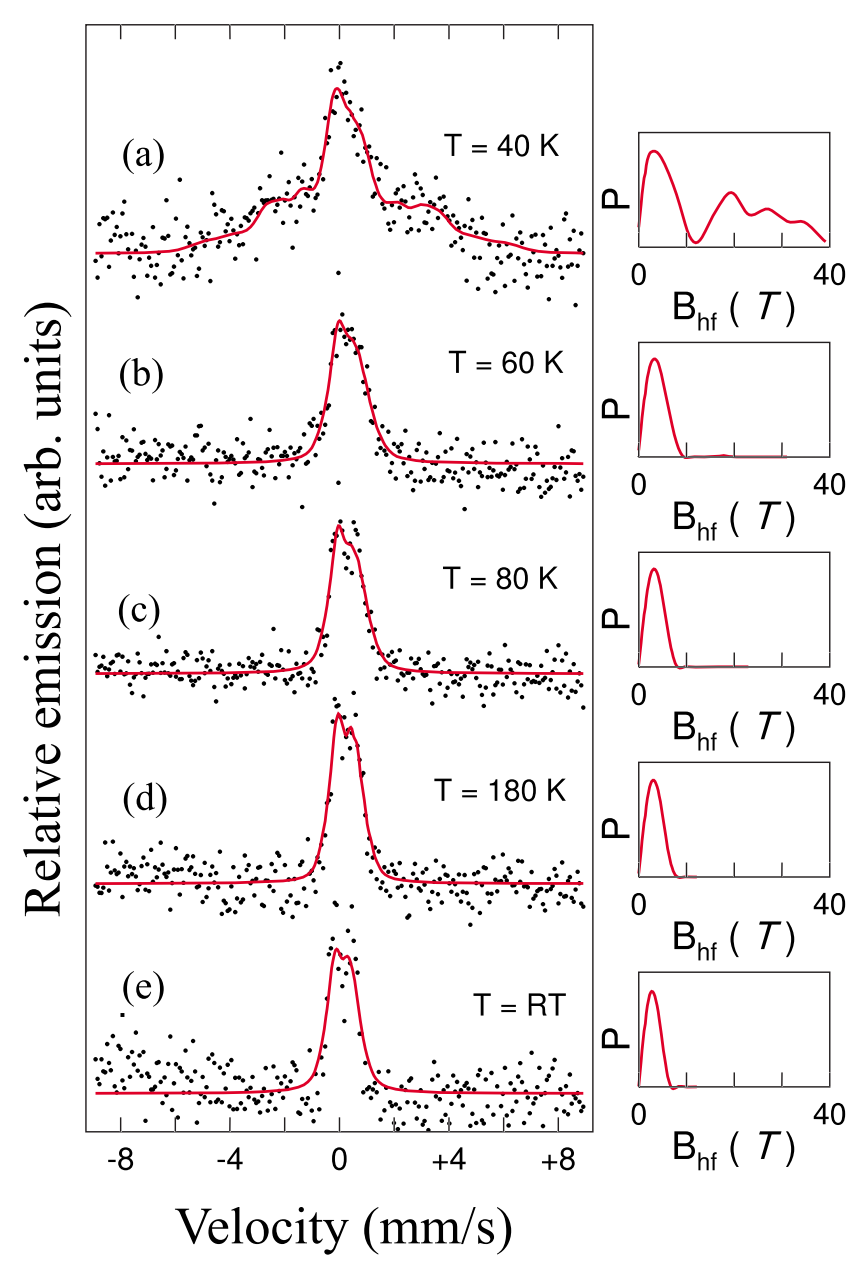

FIG. 6. (Color online) CEM spectra and hyperfine-field distributions $P\left(B_{h f}\right)$ (right) of a discontinuous ${ }^{57} \mathrm{Fe}(001)$ film of $1.9 \mathrm{ML}$ coverage grown on $\mathrm{GaAs}(001)-(4 \times 6)$ and measured in zeroexternal field in situ in UHV at a temperature of (a) $40 \mathrm{~K}$, (b) $60 \mathrm{~K}$, (c) $80 \mathrm{~K}$, (d) $180 \mathrm{~K}$, and (e) room temperature.

freezing of the superparamagnetic relaxations. It is remarkable that the low- $T$ spectra measured do not show sharp lines but are smeared out, very likely due to a distribution of various properties such as cluster sizes, relaxation times, hyperfine magnetic fields, superparamagnetic blocking temperatures $T_{B}$, and Curie temperatures. Therefore, the detailed theoretical description of the measured spectra in Figs. 6-8 is a difficult task.

In order to determine the average superparamagnetic blocking temperature and the average hyperfine field from the spectra, it is sufficient to least-squares fit all spectra in Figs. 6-8 with a magnetic hyperfine-field distribution $P\left(B_{h f}\right)$. For this fitting we assumed in-plane Fe magnetic moments, implying a line intensity ratio in the basic Zeeman sextets of distribution of $I_{23}=I_{54}=4.0$ [where $I_{23}\left(I_{54}\right)$ is the line intensity ratio of line 2 (line 5) to line 3 (line 4)]. The $P\left(B_{h f}\right)$ distributions obtained from the fitting are shown in Figs. 6-8 (right-hand side).

At RT, the real CEM spectra of the superparamagnetic Fe clusters consist of the quadrupole-split doublets described in Sec. III A 3). However, in order to obtain a quantitative mea- 


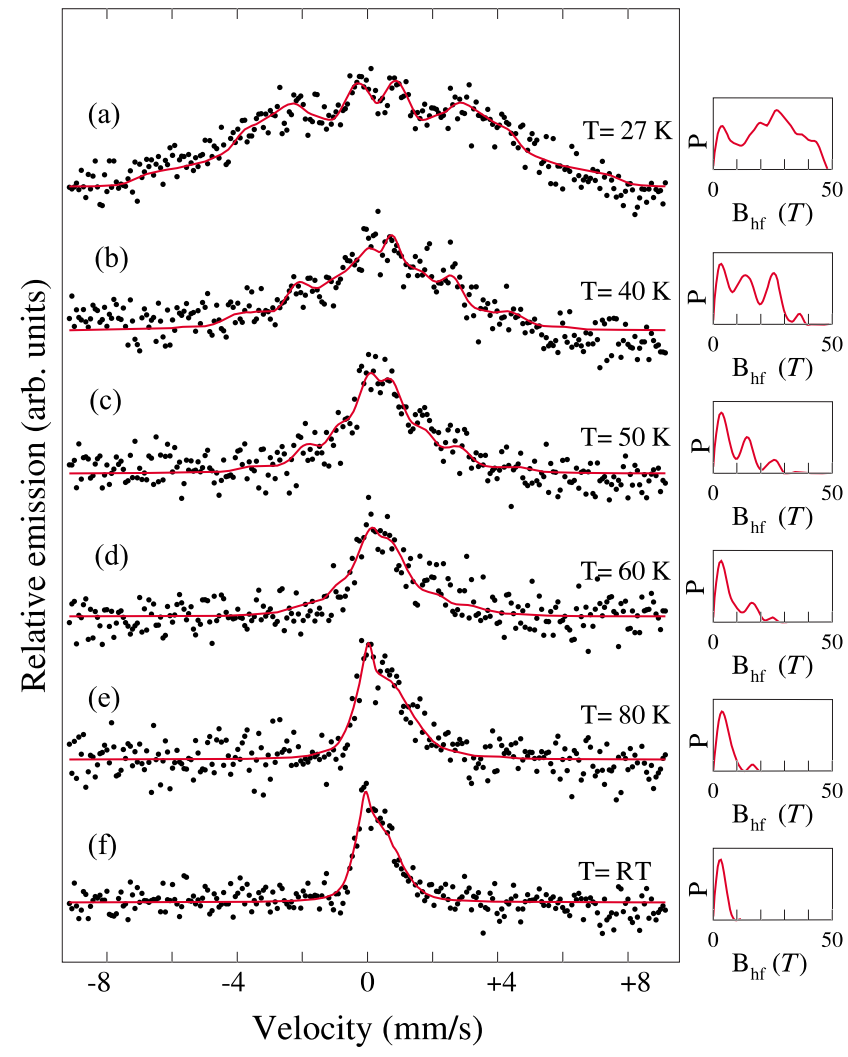

FIG. 7. (Color online) CEM spectra and hyperfine-field distributions $P\left(B_{h f}\right)$ (right) of a discontinuous ${ }^{57} \mathrm{Fe}(001)$ film of $2.2 \mathrm{ML}$ coverage grown on $\mathrm{GaAs}(001)-(4 \times 6)$ and measured in zero external field in situ in UHV at a temperature of (a) $27 \mathrm{~K}$, (b) $40 \mathrm{~K}$, (c) $50 \mathrm{~K}$, (d) $60 \mathrm{~K}$, (e) $80 \mathrm{~K}$, and (f) room temperature.

sure of the extra spectral broadening and of the average hyperfine field causing this broadening, we have consistently fitted all spectra in Figs. 6-8 (including those at RT) with a distribution $P\left(B_{h f}\right)$. The fictitious $P\left(B_{h f}\right)$ distributions of the Fe clusters at RT [bottom spectra in Figs. 6-8] show a dominant peak near a zero hyperfine field, representing the superparamagnetic state. With decreasing $T$, the hyperfine-field distributions become systematically broader. ${ }^{74}$

Figure 9(a) displays the $T$ dependence of the root-meansquare (rms) width of the distribution $P\left(B_{h f}\right)$ for ${ }^{57} \mathrm{Fe} / \mathrm{GaAs}$ at ${ }^{57} \mathrm{Fe}$ coverages of $1.9 \mathrm{ML}$ (triangles), 2.2 ML (full circles), and 2.5 ML (full squares). The data points have been obtained from Figs. 6-8. More precisely, we have plotted the excess rms width (or corrected rms width) relative to the rms width of the distribution at RT for each sample, where we know the Fe clusters to be superparamagnetic. (At RT, rms $=2.8,3.1$, and $3.2 \mathrm{~T}$ for $1.9,2.2$, and $2.5 \mathrm{ML} \mathrm{Fe}$, respectively). One can notice that upon cooling from RT, the rms width first increases only very weakly, but then shows a remarkably abrupt and drastic rise at a certain temperature specific for each sample. We identify this temperature as the superparamagnetic blocking temperature $T_{B}$ of the different samples. ${ }^{75}$ From the intersections of the linear fittings to the data points [dashed lines in Fig. 9(a)], we infer blocking temperatures of $62 \pm 5$ and $165 \pm 5 \mathrm{~K}$ for ${ }^{57} \mathrm{Fe}$ coverages of 1.9 and $2.5 \mathrm{ML}$, respectively. For $2.2 \mathrm{ML}{ }^{57} \mathrm{Fe}$ coverage, the

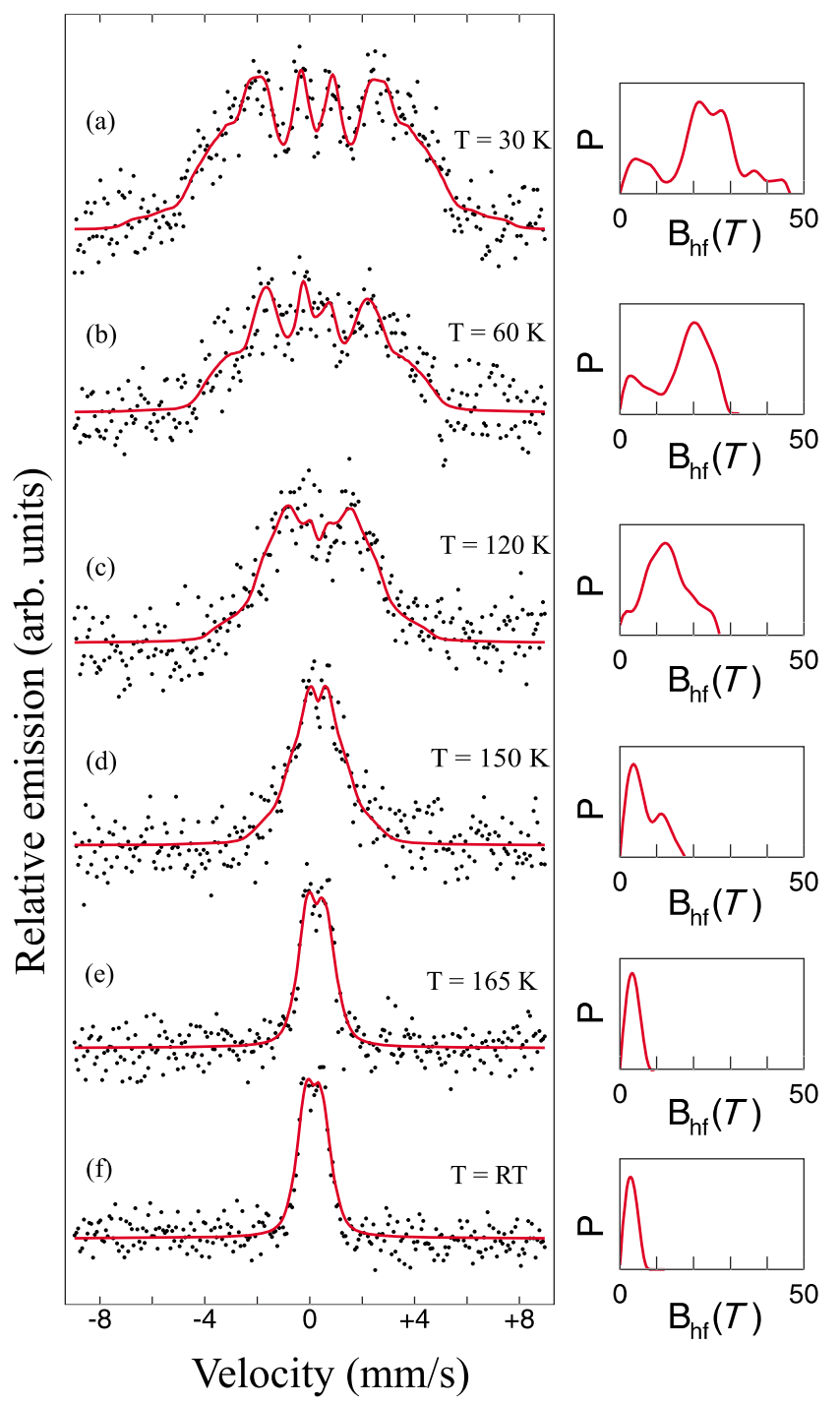

FIG. 8. (Color online) CEM spectra and hyperfine-field distributions $P\left(B_{h f}\right)$ (right) of a discontinuous ${ }^{57} \mathrm{Fe}(001)$ film of $2.5 \mathrm{ML}$ coverage grown on $\mathrm{GaAs}(001)-(4 \times 6)$ and measured in zeroexternal field in situ in UHV directly after deposition at a temperature of (a) $30 \mathrm{~K}$, (b) $60 \mathrm{~K}$, (c) $120 \mathrm{~K}$, (d) $150 \mathrm{~K}$, (e) $165 \mathrm{~K}$, and (f) room temperature.

transition appears to be less sharp and more rounded, starting as low as $\sim 80 \mathrm{~K}$ upon cooling. We estimate $T_{B}$ as $80 \pm 10 \mathrm{~K}$.

The $T$ dependence of the average hyperfine field $\left\langle B_{h f}\right\rangle$ of the different samples is shown in Fig. 9(b). The data points have been obtained from the $P\left(B_{h f}\right)$ distributions shown in Figs. 6-8. To be more precise, in Fig. 9(b) we have plotted the excess average hyperfine field (or corrected average $\left.\left\langle B_{h f}\right\rangle\right)$ relative to the (fictitious) average hyperfine field for each sample at RT, where the Fe clusters are superparamagnetic. (This fictitious average hyperfine field at RT was found to be 3.1, 3.6, and 3.2 T for 1.9, 2.2, and 2.5 ML Fe, respectively.)

A comparison of Figs. 9(a) and 9(b) shows that the $T$ dependence of the corrected rms and the corrected $\left\langle B_{h f}\right\rangle$ is very similar. Again, we identify the superparamagnetic blocking temperature as the temperature at which the hyper- 


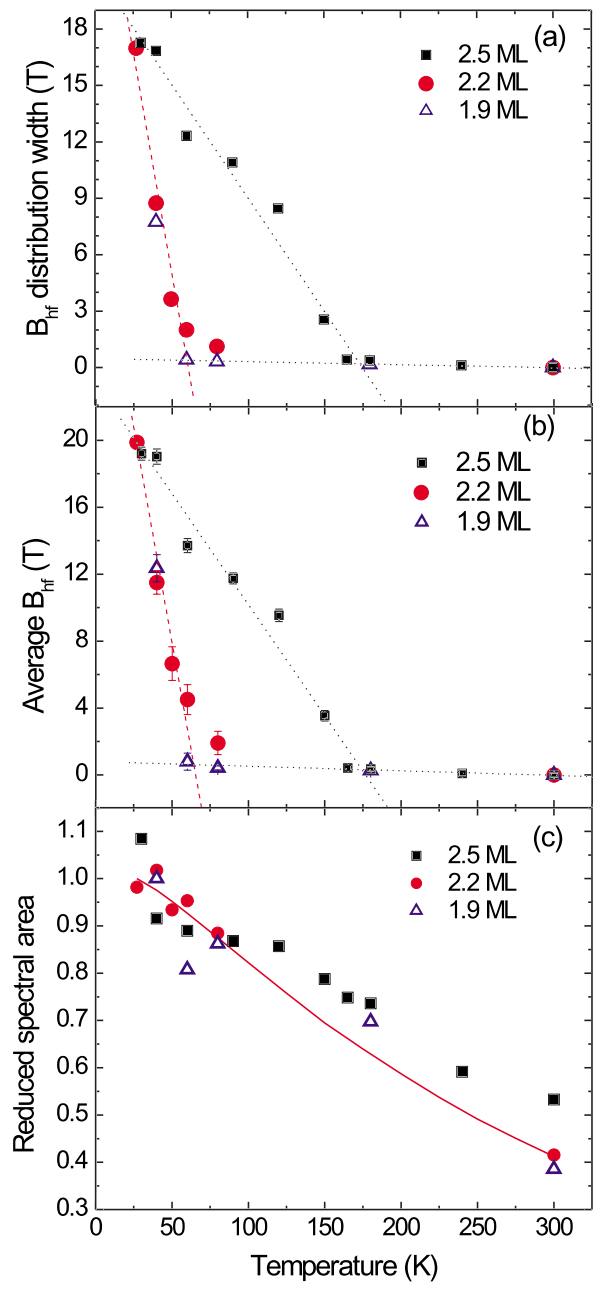

FIG. 9. (Color online) Temperature dependence of (a) measured excess root-mean-square width of the hyperfine-field distribution $P\left(B_{h f}\right)$ (relative to that at RT), (b) measured excess average magnetic hyperfine field $\left\langle B_{h f}\right\rangle$ (relative to that at room temperature), and (c) measured reduced spectral area. The ${ }^{57} \mathrm{Fe}(001)$ coverages are 2.5 ML (full squares), 2.2 ML (full circles), and 1.9 ML (open triangles). The full-drawn line in (c) shows the normalized DebyeWaller factor ( $f$ factor) (normalized by the average $f$ value between 27 and $40 \mathrm{~K}$ ) calculated according to the Debye model for $\Theta_{D}$ $=196 \mathrm{~K}$. All measured quantities were obtained from Figs. 6-8. The dotted lines in (a) and (b) are a guide for the eye.

fine field falls to zero. ${ }^{75}$ From the intersections of linear fittings to the data points in Fig. 9(b), we obtain blocking temperatures of $62 \pm 5$ and $165 \pm 5 \mathrm{~K}$ for 1.9 and $2.5 \mathrm{ML}{ }^{57} \mathrm{Fe}$ coverages, respectively, in agreement with the $T_{B}$ values inferred from Fig. 9(a). According to the data points for 2.2 ML coverage, the transition for this sample is again found to be less sharp and appears to start at $\sim 80 \mathrm{~K}$ upon cooling, with $T_{B}$ estimated to be $80 \pm 10 \mathrm{~K}$.

\section{Superparamagnetism}

We now return to the question whether our uncoated ${ }^{57} \mathrm{Fe}$ nanoclusters on GaAs(001)- $(4 \times 6)$ in UHV show superparamagnetic behavior with a blocking temperature $T_{B}$ or whether the temperature labeled $T_{B}$ in reality is a critical temperature (magnetic ordering temperature $T_{C}$ ) indicative of a ferromagnetic phase transition. Bensch et al. ${ }^{32}$ have argued that the onset of ferromagnetism below a certain temperature at a certain critical Fe coverage in the ultrathin regimes of $\mathrm{Fe} / \mathrm{GaAs}(001)-(4 \times 2)$ and $(2 \times 6)$ is related to a two-dimensional (2D) phase transition. Based on MOKE measurements performed ex situ on an Au-coated wedgeshaped Fe film, Bensch et al $^{32}$ could describe the $T$ dependence of the remanent Kerr rotation by a power law $M_{S}$ $\propto\left(1-T / T_{C}\right)^{\beta}$ with a critical exponent $\beta=0.26$, as expected for $2 \mathrm{D} X Y$ systems of finite size $\left(M_{S}=\right.$ spontaneous magnetization). However, our zero-field in situ CEMS results in $\mathrm{UHV}$ on single-layer Fe films on $\mathrm{GaAs}(001)-(4 \times 6)$ are at odds with the findings of Bensch et al. The striking quasilinear temperature dependence of the average hyperfine field $\left\langle B_{h f}\right\rangle$ observed for $T \leqslant T_{B}$ on our ultrathin uncapped Fe films by CEMS [Fig. 9(b)] is not characteristic of a magnetic phase transition but rather provides strong evidence for a superparamagnetic transition. Under no circumstances can our data points for 2.5 and 2.2 $\mathrm{ML}^{57} \mathrm{Fe}$ coverage, [Fig. 9(b)] be described by the power law of a $2 \mathrm{D}$ (or 3D) magnetic phase transition. For 1.9 ML Fe coverage, we do not have enough data points to give a conclusive answer, but the data points are consistent with those at 2.2 ML coverage.

Our interpretation of the quasilinear $T$ dependence of $\left\langle B_{h f}\right\rangle$ in terms of a superparamagnetic transition is strongly supported by ex situ superconducting quantum interference device (SQUID) measurements of the remanent magnetization $M_{R}$ versus $T$ of Al-coated Fe/GaAs(001) films at 3.03.5 ML Fe coverage (i.e., just below the critical coverage for coalescence), reported by Herfort et al. ${ }^{48}$ One can notice in Fig. 4 of that work ${ }^{48}$ that $M_{R}(T)$ follows approximately a linear $T$ dependence below the transition temperature, whereby the range of this linear behavior increases by reducing the Fe thickness from 3.5 to 3.0 ML. Simultaneously, the magnetic ordering temperature was observed to decrease with decreasing Fe coverage. ${ }^{48}$ This behavior can be understood by the nucleation and growth of Fe islands at the initial stages of growth. With decreasing Fe coverage, away from the critical value for coalescence, the fraction of isolated Fe clusters in the film, which are superparamagnetic, increases. This leads to the appearance of the approximately linear sections of $M_{R}(T)$ below the ordering temperature, with the linear range being more extended if the Fe coverage is further below the thickness for coalescence. Our interpretation of the quasilinear $\left\langle B_{h f}\right\rangle$ versus $T$ behavior in terms of a superparamagnetic transition is also supported by SQUID measurements of $M_{R}(T)$ of superparamagnetic epitaxial ultrathin magnetic $\left(\mathrm{Fe}_{3} \mathrm{O}_{4}\right)$ films by Voogt et al. ${ }^{76}$ These authors observed a nearly linear increase of $M_{R}$ with decreasing $T$ below the blocking temperature, similar to our observation in Fig. 9(b). This rise of $M_{R}$ with decreasing $T$ is governed by the relaxation time $\tau$, i.e., the time to reverse the magnetic domain (or cluster) magnetization over an energy barrier of height $W{ }^{76}$ An approximately linear increase of the measured magnetization upon cooling below $T_{B}$ was reported also by Chien ${ }^{77}$ and Xiao et al. ${ }^{78}$ in their work on $\mathrm{Fe}-\left(\mathrm{SiO}_{2}\right)$ granular films. Thus, there is convincing evidence for a quasilinear $T$ dependence of $M_{R}$ below $T_{B}$ in various superpara- 
magnetic systems. As the ${ }^{57} \mathrm{Fe}$ hyperfine magnetic field $B_{h f}$ is proportional to the spontaneous magnetization ${ }^{79}$ (or to the remanent magnetization in a single magnetic domain), we can expect a quasi-linear $B_{h f}$ versus $T$ behavior below $T_{B}$, as experimentally observed in our Fig. 9(b) for ${ }^{57} \mathrm{Fe}$ nanoclusters on $\operatorname{GaAs}(001)$. The superparamagnetic behavior in $\mathrm{Fe} / \mathrm{GaAs}(001)-(4 \times 6)$ at low coverages, i.e., below the critical thickness $d_{C} \approx 4$ ML Fe at which ferromagnetism occurs, was also inferred from in situ Brillouin light scattering by Steinmüller et al. ${ }^{45}$

The superparamagnetic relaxation of the magnetization in noninteracting single-domain particles in the zero applied field can be described by the Arrhenius law for the relaxation time $\tau^{73,77,80}$

$$
\tau=\tau_{0} e^{C V / k_{B} T},
$$

where $k_{B}$ is the Boltzmann constant, $\tau_{0}^{-1}$ is the attempt frequency $\left(\sim 10^{11} \mathrm{~s}^{-1}\right), C$ is the magnetic anisotropy constant, $V$ is the cluster volume, $T$ is the temperature, and $C V=W$ is the magnetic anisotropy energy. The observed $T_{B}$ depends on the characteristic time window of the measurement technique, $\tau_{m}$. For $\tau \ll \tau_{m}$, the system behaves superparamagnetically, and for $\tau \gg \tau_{m}$, the system is in the blocked (frozen) state. $T_{B}$ is then defined by $\tau=\tau_{m}$. From

$$
T_{B}=\frac{C V}{k_{B}\left[\ln \left(\tau_{m} / \tau_{0}\right)\right]},
$$

one can determine the magnetic anisotropy energy $(\mathrm{CV})$ of the clusters using the characteristic Mössbauer observation time $\tau_{m} \approx 10^{-8} \mathrm{~s}\left({ }^{57} \mathrm{Fe}\right.$ nuclear Larmor precession time), the measured $T_{B}$ values, and choosing $\tau_{0} \approx 10^{-11} \mathrm{~s}^{76}$

By using our measured $T_{B}$ values of 62,80 , and $165 \mathrm{~K}$ for 1.9, 2.2, and 2.5 ML Fe coverage, respectively, the following values for the anisotropy energy $(C V)$ are obtained: 5.9 $\times 10^{-14} \mathrm{erg}(37 \mathrm{meV})$ for $1.9 \mathrm{ML}, 7.6 \times 10^{-14} \mathrm{erg}(48 \mathrm{meV})$ for $2.2 \mathrm{ML}$, and $15.7 \times 10^{-14} \mathrm{erg}(98 \mathrm{meV})$ for $2.5 \mathrm{ML}{ }^{57} \mathrm{Fe}$ coverage. These values, obtained from our ${ }^{57} \mathrm{Fe} / \mathrm{GaAs}$ nanoclusters, are of the same order of magnitude as the anisotropy energy obtained by Chien ${ }^{77}$ and Xiao et al. ${ }^{78}$ on $\mathrm{Fe}_{50}\left(\mathrm{SiO}_{2}\right)_{50}$ nanoparticles of $2.5 \mathrm{~nm}$ average diameter, i.e., $C V \approx 8.2$ $\times 10^{-14} \mathrm{erg}$.

The average volume $V$ of the ${ }^{57} \mathrm{Fe}$ nanoclusters in our samples has been obtained from our STM results (Sec. III A 2). The average values obtained by STM are 1.84, 20.03, 25.20, and $30.47 \mathrm{~nm}^{3}$ for Fe coverages of 1, 2.5, 3, and 4 ML, respectively [Fig. 2(e)]. By interpolation, we obtain a cluster volume of $11-12.75 \mathrm{~nm}^{3}$ at $1.9 \mathrm{ML}$ coverage and $15-16.5 \mathrm{~nm}^{3}$ at $2.2 \mathrm{ML}$ coverage. This allows us to calculate the magnetic anisotropy constant $C$ in our samples. The $C$ values derived are $(4.6-5.4) \times 10^{5} \mathrm{~J} / \mathrm{m}^{3}$ for our 1.9 2.2 ML Fe samples and $7.8 \times 10^{5} \mathrm{~J} / \mathrm{m}^{3}$ for our $2.5 \mathrm{ML} \mathrm{Fe}$ sample.

\section{Discussion of magnetic anisotropy and blocking temperature}

Our $C$ values should be compared with literature values of the effective uniaxial magnetic anisotropy constant $K_{U}^{\text {eff }}$ $=K_{U}^{V}+K_{U}^{\text {int }} / t_{\mathrm{Fe}}$ for Ga-terminated $\mathrm{Fe} / \mathrm{GaAs}(001)$, as in our case. Here, $K_{U}^{V}$ is the volume contribution and $K_{U}^{\text {int }}$ is the interface term that dominates for $t_{\mathrm{Fe}}<10 \mathrm{ML}{ }^{27}$ Therefore, we may compare our $C$ values with the effective uniaxial anisotropy $K_{U}^{\text {eff }} \approx K_{U}^{\text {int }} / t_{\mathrm{Fe}}$ reported in the literature. For the Ga-terminated case, Herfort et al..$^{48}$ performed SQUID magnetometry on Al-coated samples and obtained $K_{U}^{\text {eff }}=0.87$ $\times 10^{5} \mathrm{~J} / \mathrm{m}^{3}$ at RT for $t_{\mathrm{Fe}}=5 \mathrm{ML}$. At low temperature $(T=10 \mathrm{~K})$, Herfort et al. ${ }^{48}$ obtained $K_{U}^{\text {eff }}=2.2 \times 10^{5} \mathrm{~J} / \mathrm{m}^{3}$ for $t_{\mathrm{Fe}}=4 \mathrm{ML}, K_{U}^{\text {eff }}=0.5 \times 10^{5} \mathrm{~J} / \mathrm{m}^{3}$ for $t_{\mathrm{Fe}}=3 \mathrm{ML}$, and $K_{U}^{\text {eff }} \approx 0$ for $t_{\mathrm{Fe}}=2.5 \mathrm{ML}$. Apparently, $K_{U}^{\text {eff }}$ decreases to about zero (at $2.5 \mathrm{ML}$ ) with decreasing $\mathrm{Fe}$ coverage. This is surprising since $K_{U}^{e f f}$ is dominated by the interface term $K_{U}^{i n t} / t_{\mathrm{Fe}}$, which should increase with decreasing $t_{\mathrm{Fe}}$. The authors ${ }^{48}$ regarded this strong reduction of $K_{U}^{\text {eff }}$ below $t_{\mathrm{Fe}}=4 \mathrm{ML}$ (which is accompanied by a strong reduction of the saturation magnetization $M_{S}$ ) as an indication that the structural configuration of the $\mathrm{Fe} / \mathrm{GaAs}$ interface is not entirely established before the complete coalescence of the Fe islands occurs. Our observation of superparamagnetism for uncoated $\mathrm{Fe} / \mathrm{GaAs}(001)$ at $2.5 \mathrm{ML}$ coverage, together with the high magnetic anisotropy value $C$ of $\sim 7.8 \times 10^{5} \mathrm{~J} / \mathrm{m}^{3}$ obtained for this film, is in disagreement with the result of Herfort $e t$ al. on Al-coated Fe/GaAs. We attribute this discrepancy to intermixing and $\mathrm{Fe}-\mathrm{Al}$ interfacial alloy formation in the $\mathrm{Al}-$ coated ultrathin $\mathrm{Fe} / \mathrm{GaAs}$ films used in Ref. 48 since the formation of interfacial $\mathrm{Fe}-\mathrm{Al}$ solid solutions and intermetallic compounds has been clearly observed in roomtemperature deposited $\mathrm{Fe} / \mathrm{Al}$ multilayers. ${ }^{81,82} \mathrm{Fe}-\mathrm{Al}$ interface alloying will result in a strong reduction of $M_{S}$ and $K_{U}^{\text {int }}$ (and $K_{U}^{e f f}$ ), particularly at ultrathin Fe coverages of $t_{\mathrm{Fe}} \leqslant 2.5 \mathrm{ML}$, where our uncoated (free) Fe clusters still show magnetic ordering and large anisotropy at low $T$.

Moosbühler et al $^{33}$ have employed ex situ MOKE to determine $K_{U}^{\text {eff }}$ for Au-coated epitaxial Fe films on Gaterminated $(4 \times 2)$ and $(2 \times 6) \mathrm{GaAs}(001)$ surfaces as a function of Fe coverage at RT. According to Fig. 4 of Ref. 33, $K_{U}^{e f f} \approx 12 \times 10^{5} \mathrm{erg} / \mathrm{cm}^{3}\left(\approx 1.2 \times 10^{5} \mathrm{~J} / \mathrm{m}^{3}\right)$ at $t_{\mathrm{Fe}}=5 \mathrm{ML}$ and drops to $K_{U}^{e f f} \approx 10 \times 10^{5} \mathrm{erg} / \mathrm{cm}^{3}\left(\approx 1 \times 10^{5} \mathrm{~J} / \mathrm{m}^{3}\right)$ at $t_{\mathrm{Fe}}=4$ ML for both types of surface reconstructions. These $K_{U}^{\text {eff }}$ values, measured at RT, are smaller than our $C$ values $\left[C \approx(4.6-5.4) \times 10^{5} \mathrm{~J} / \mathrm{m}^{3}\right.$ for $t_{\mathrm{Fe}}=1.9-2.2 \quad \mathrm{ML}$ and $C \approx 7.8 \times 10^{5} \mathrm{~J} / \mathrm{m}^{3}$ for $\left.t_{\mathrm{Fe}}=2.5 \mathrm{ML}\right]$ obtained by Mössbauer spectroscopy at low temperatures and at lower Fe coverages, but are of the same order of magnitude. Since magnetic anisotropies usually increase by reducing $T$, enhanced $K_{U}^{\text {eff }}$ values are expected at lower temperatures. Moreover, $K_{u}^{e f f}$ is expected to increase at lower Fe coverages because of the interface term $K_{U}^{i n t} / t_{\mathrm{Fe}}$. Surprisingly, this expected behavior was not observed in the low-temperature magnetometric measurements on Au-coated $\mathrm{Fe} / \mathrm{GaAs}(001)$ by Bensch et $a l .3^{34}$ At $10 \mathrm{~K}, K_{U}^{\text {int }}=K_{U}^{e f f} \cdot t_{\mathrm{Fe}}$ was found to drop from $\approx 0.04 \mathrm{erg} / \mathrm{cm}^{2}$ (or $K_{U}^{\text {eff }} \approx 0.90 \times 10^{5} \mathrm{~J} / \mathrm{m}^{3}$ ) at $t_{\mathrm{Fe}}=3.1 \mathrm{ML}$ to zero $\left(K_{U}^{\text {eff }} \approx 0\right)$ at $t_{\mathrm{Fe}}=2.5 \mathrm{ML}$. The disappearance of $K_{U}^{\text {eff }}$ at and below $t_{\mathrm{Fe}}=2.5 \mathrm{ML}$ for Au-coated Ga-terminated $\mathrm{Fe} / \mathrm{GaAs}(001)$ at low $T$, as observed by Bensch et al. ${ }^{34}$ agrees with the low- $T$ result reported by Herfort $e t$ al. ${ }^{48}$ for Al-coated $\mathrm{Fe} / \mathrm{GaAs}(001)$ samples. However, both results are in striking contrast to our Mössbauer results, which clearly demonstrate, via the appearance of the hyperfine magnetic field $\left\langle B_{h f}\right\rangle$ at low $T$, that uncoated (free) Fe clusters on Ga- 
terminated $\mathrm{GaAs}(001)$ surfaces at Fe coverages $t_{\mathrm{Fe}} \leqslant 2.5 \mathrm{ML}$ are intrinsically magnetically ordered and behave superparamagnetically above $T_{B}$, implying a large effective anisotropy constant $C$ (or $K_{U}^{\text {eff }}$ ). Moreover, our results on uncoated $\mathrm{Fe} / \mathrm{GaAs}$ films unambiguously demonstrate that contrary to the case of Al-coated ${ }^{48}$ and $\mathrm{Au}$-coated ${ }^{32-34}$ films, the intrinsic ferromagnetic order within the uncoated Fe clusters does not break down at and below 2.5 ML Fe coverage; this is clearly proven by the persistence of the hyperfine magnetic field $\left\langle B_{h f}\right\rangle$ at low $T$ for $t_{\mathrm{Fe}} \leqslant 2.5 \mathrm{ML}$ in uncoated films. We attribute the discrepancy between the results by Bensch et al. ${ }^{34}$ and our findings to the influence of the Au coating layer used in Ref. 34. In fact, for the growth of $\mathrm{Au}$ on $\mathrm{Fe}(001)$ at low temperature $(370 \mathrm{~K})$, a surface Au-Fe alloy was observed by STM at submonolayer Au coverage. ${ }^{83,84}$ This surfaceconfined alloy demixes when it is further covered with $\mathrm{Au}$, and in combination with layer-by-layer growth, a rough interface consisting of $\mathrm{Au}$ islands and $\mathrm{Fe}$ islands on the original $\mathrm{Fe}(001)$ substrate is created. ${ }^{83,84}$ Apparently, such a severe $\mathrm{Au}-\mathrm{Fe}$ surface alloying affects the magnetic properties of ultrathin $\mathrm{Fe}$ layers (saturation magnetization $M_{S}$ and uniaxial interface magnetic anisotropy $K_{U}^{\text {int }}$ ), particularly at very low Fe coverages, such as $t_{\mathrm{Fe}} \leqslant 2.5 \mathrm{ML}$, and leads to $M_{S} \approx 0$ and $K_{U}^{\text {int }} \approx 0$ at low temperatures $(T \rightarrow 0 \mathrm{~K})$. By contrast, our uncoated free $\mathrm{Fe}$ nanoclusters on GaAs manifest the real intrinsic properties of this system, unaffected by surface-coating materials.

The only literature report on uncoated (free) $\mathrm{Fe} / \mathrm{GaAs}(001)-(4 \times 6)$ ultrathin films in UHV at low temperature is that of Zakeri et al. ${ }^{53}$ These researchers performed in situ ferromagnetic resonance (FMR) studies in UHV in the temperature range of $40 \mathrm{~K} \leqslant T \leqslant 400 \mathrm{~K}$ at $\mathrm{Fe}$ coverages between 5-20 ML. Due to experimental limitations, they could measure only the perpendicular component of the anisotropy constant. They reported a strong perpendicular surface anisotropy contribution of $K_{2 \perp}^{s, e f f}=1.26$ $\times 10^{-3} \mathrm{~J} / \mathrm{m}^{2}$ and a smaller volume contribution of $K_{2 \perp}^{v}$ $=(4 \pm 9) \times 10^{4} \mathrm{~J} / \mathrm{m}^{3}$ (both values obtained from extrapolation to $T=0 \mathrm{~K})$. For the thinnest $\mathrm{Fe}$ coverage $\left(t_{\mathrm{Fe}}=5 \mathrm{ML}\right)$ at $40 \mathrm{~K}$, one can estimate a value of $K_{2 \perp}^{\text {eff }} \approx 16 \times 10^{5} \mathrm{~J} / \mathrm{m}^{3}$ from the insert in Fig. 4 of Ref. 53. This value, obtained by FMR at low $T$, is of the same order of magnitude as our anisotropy values of $C \approx 5 \times 10^{5} \mathrm{~J} / \mathrm{m}^{3}$ (at $t_{\mathrm{Fe}}=1.9-2.2 \mathrm{ML}$ ) and $C$ $\approx 7.8 \times 10^{5} \mathrm{~J} / \mathrm{m}^{3}$ (at $t_{\mathrm{Fe}}=2.5 \mathrm{ML}$ ), also determined on uncoated (free) $\mathrm{Fe} / \mathrm{GaAs}(001)-(4 \times 6)$ in UHV at low temperatures. The appearance of an out-of-plane anisotropy component agrees with theoretical predictions by Košuth et al. ${ }^{85}$ who found that a part of the magnetic anisotropy energy favors an out-of-plane orientation for $\mathrm{GaAs}(001) / \mathrm{Fe}_{n}$ $(n=1-7 \mathrm{ML})$.

Moreover, large effective perpendicular (out-of-plane) surface/interface anisotropy constants were observed at RT by McPhail et $a l^{42}$ on Au-coated 4.1 ML thick $\mathrm{Fe} / \mathrm{GaAs}(001)-(4 \times 6)$ by ex situ polar MOKE measurements $\left(\left|K_{p}^{e f f}\right|=15 \times 10^{5} \mathrm{~J} / \mathrm{m}^{3}\right)$ and by Madami et al. ${ }^{47}$ on uncoated $5 \mathrm{~A}$ thick $(3.5 \mathrm{ML}) \mathrm{Fe} / \mathrm{GaAs}(001)-(4 \times 6)$ by in situ Brillouin light scattering $\left(K_{\text {out }}^{\text {eff }}=K_{\text {out }}^{s} / t_{\mathrm{Fe}}=8.0 \times 10^{5} \mathrm{~J} / \mathrm{m}^{3}\right)$. Some of the measured $K_{2 \perp}^{\text {eff }}=K_{\text {out }}^{\text {eff }}$ values are larger than the demagnetization energy $2 \pi M_{S}^{2}=16.1 \times 10^{5} \mathrm{~J} / \mathrm{m}^{3}$ of Fe, forc- ing the magnetization in the film plane. However, the strong uniaxial in-plane surface anisotropy has to be subtracted from these $K_{2 \perp}^{\text {eff }}$ values, reducing them to values below $2 \pi M_{S}^{2}$ and leaving the magnetization in the film plane. These values are of the same order of magnitude as our $C$ values. It is interesting in this context that strong effective perpendicular anisotropy constants $K_{2 \perp}$ of $14.6 \times 10^{5} \mathrm{~J} / \mathrm{m}^{3}$ at $t_{\mathrm{Fe}}=4 \mathrm{ML}$ and $2.1 \times 10^{5} \mathrm{~J} / \mathrm{m}^{3}$ at $t_{\mathrm{Fe}}=33 \mathrm{ML}$ were also determined by ferromagnetic resonance for the case of epitaxial $\mathrm{Fe}$ layers on GaAs(001) surfaces that were As capped (and As desorbed in UHV) before Fe deposition. ${ }^{44}$ This desorption of the As cap layer usually leads to an As-terminated GaAs(001) surface, ${ }^{52}$ but the magnetic anisotropy is known to be fairly independent of the $\mathrm{GaAs}(001)$ surface reconstruction..$^{33,48}$ These large $K_{2 \perp}$ values $^{44}$ are in qualitative agreement with our anisotropy $(C)$ values obtained by Mössbauer spectroscopy. However, since Zhai et al. ${ }^{44}$ gave neither their measurement temperature nor the type of protective coating material, we cannot draw further conclusions from this comparison.

We now compare our superparamagnetic blocking temperatures $T_{B}$ (measured in zero-external field in situ in UHV on uncoated Fe films) with magnetic transition temperatures for $\mathrm{Fe} / \mathrm{GaAs}(001)$ reported in the literature. Unfortunately, with one exception, we can compare only with Au-coated or $\mathrm{Al}-$ coated $\mathrm{Fe}$ films reported in the literature. Bensch et al. ${ }^{32}$ measured the Fe-thickness dependence of the transition temperatures $T_{C}$ by ex situ MOKE on Au-coated wedge-shaped Fe films at Fe coverages between $2.8 \leqslant t_{\mathrm{Fe}} \leqslant 3.6 \mathrm{ML}$. They interpreted their $T_{C}$ as the magnetic ordering temperature of a 2D system and could describe $T_{C}$ vs $t_{\mathrm{Fe}}$ by a finite-size scaling law, which (extrapolated to $T_{C}=0 \mathrm{~K}$ ) resulted in a critical thickness of $t_{\mathrm{Fe}}=2.5 \mathrm{ML}$ for the onset of ferromagnetism. ${ }^{32}$ However, the lowest measurement temperature in Ref. 32 was only about 100 K. Keeping Eq. (3) in mind, we can predict from our superparamagnetic blocking temperature $T_{B}$ (Möss) (measured by CEMS) the blocking temperature $T_{B}$ (MOKE/SQUID) expected from a magnetometric MOKE (and SQUID) measurement on the same sample by using the ratio defined in Ref. 77,

$$
\frac{T_{B}(\text { MOKE/SQUID })}{T_{B}(\mathrm{Möss})}=\frac{\ln \left(\tau_{2} / \tau_{0}\right)}{\ln \left(\tau_{1} / \tau_{0}\right)} .
$$

Here, $\tau_{1} \approx 10-100 \mathrm{~s}$ is the characterization observation time in MOKE (and SQUID) measurements, ${ }^{76,77}$ and $\tau_{2}=\tau_{m}$ $\approx 10^{-8}$ in a Mössbauer measurement. For an ac-MOKE measurement (as performed by Bensch et al. ${ }^{32}$ ), $\tau_{1} \approx 10^{-2} \mathrm{~s}$. The predicted values of $T_{B}$ (MOKE/SQUID) range from 14-22, $18-28$, and $38-58 \mathrm{~K}$ for our samples with $t_{\mathrm{Fe}}=1.9,2.2$, and 2.5 ML, respectively. In the case of ac-MOKE, the predicted values are about 20,26 , and $54 \mathrm{~K}$ for analogous Fe thicknesses. None of these temperatures were accessible in the work by Bensch et al. ${ }^{32}$ Ploog et al. ${ }^{40}$ mentioned a $T_{B}$ value of $30 \mathrm{~K}$ at $2.5 \mathrm{ML} \mathrm{Fe}$ in their work; however, below $2.5 \mathrm{ML}$, these researchers did not observe a magnetic signal, contrary to what is obtained from our Mössbauer results on uncoated $\mathrm{Fe} / \mathrm{GaAs}$ films. This discrepancy may be explained by Fe-Al intermixing due to the $\mathrm{Al}$ coating layer used in that work. 
Similarly, in the low-temperature SQUID work by Bensch et $a l .{ }^{34}$ no magnetic signal was detected at $10 \mathrm{~K}$ for $t_{\mathrm{Fe}} \leqslant 2.5$ $\mathrm{ML}$, very likely due to Fe-Au intermixing with the Au coating layer. By contrast, Zakeri et al. ${ }^{86}$ reported a superparamagnetic blocking temperature of $50 \mathrm{~K}$ at approximately 2.3 ML Fe coverage in their recent in situ FMR study on uncoated $\mathrm{Fe} / \mathrm{GaAs}(001)$. This value is in fair agreement with our $T_{B}$ values of $\sim 60-80 \mathrm{~K}$, also obtained on uncoated $\mathrm{Fe}$ films of similar coverages.

\section{Lattice dynamics}

For an isotropic system with lattice vibrations in the harmonic approximation, the recoil-energy free fraction $f$ of the nuclear resonant absorption events at temperature $T$ (the Debye-Waller factor or Lamb-Mössbauer factor, or simply $f$ factor) is given by Ref. 71: $f(T)=\exp \left(-k^{2}\left\langle x^{2}\right\rangle\right)$, where $\left\langle x^{2}\right\rangle$ is the mean-square displacement of the ${ }^{57} \mathrm{Fe}$ nucleus along the $\gamma$-ray direction and $k$ is the wave vector of the $\gamma$ radiation. In the Debye model, $f$ is expressed by ${ }^{87}$

$$
f(T)=\exp \left\{-\left[\frac{3 E_{R}}{2 k_{B} \Theta_{D}}\right]\left[1+4\left(\frac{T}{\Theta_{D}}\right)^{2} \int_{0}^{\Theta / T} \frac{x}{\exp x-1} d x\right]\right\} .
$$

Here, $k_{B}$ is the Boltzmann constant, $\Theta_{D}$ is the Debye temperature, the integral is known as the Debye integral, and $E_{R}$ is the recoil energy of the free ${ }^{57} \mathrm{Fe}$ nucleus $\left(E_{R}=1.956275\right.$ $\times 10^{-3} \mathrm{eV}$ for ${ }^{57} \mathrm{Fe}$ ).

The $f$ factor is known to be proportional to the normalized spectral area $A$ of the Mössbauer spectrum (normalized by the nonresonant number of counts). ${ }^{88}$ In Fig. 9(c), we have plotted the $T$ dependence of the reduced spectral area $A(T) / A_{a v}$ of our three samples, obtained from the CEM spectra in Figs. 6-8. $\left(A_{a v}\right.$ is the average reduced spectral area, averaged over the low- $T$ values between 27 and $40 \mathrm{~K}$ ). The $T$ dependence of the reduced spectral area was calculated from Eq. (5) and least-squares fit to the experimental spectral area ratio of ${ }^{57} \mathrm{Fe} / \mathrm{GaAs}(001)$ with $2.2 \mathrm{ML} \mathrm{Fe}$ coverage [full circles in Fig. 9(c)]. From this fitting [full-drawn curve in Fig. 9(c)], we obtain a Debye temperature $\Theta_{D}$ of $196 \pm 4 \mathrm{~K}$ for the Fe nanoclusters at 2.2 ML coverage. As can be noticed in Fig. 9(c), the experimental data points for 1.9 ML Fe coverage [Fig. 9(c), triangles] are also, on the average, well described by the fit curve for $\Theta_{D}=196 \mathrm{~K}$. On the other hand, above $\sim 100 \mathrm{~K}$, the data points for the sample with $2.5 \mathrm{ML}$ Fe coverage [Fig. 9(c), full squares] are systematically higher than the fit curve, implying a somewhat larger Debye temperature than $196 \mathrm{~K}$ for this sample. The value of $\Theta_{D}$ $=196 \mathrm{~K}$ for the Fe nanoclusters is strikingly smaller than the Debye temperature of $433 \pm 8 \mathrm{~K}$ for bulk bcc-Fe. ${ }^{89}$

The softening of the lattice vibrations revealed by the drastic reduction of $\Theta_{D}$ in our Fe nanoclusters on $\operatorname{GaAs}(001)$ can be caused by two (probably superimposed) effects: (i) interfacial $\mathrm{Fe}-\mathrm{Ga}$ and/or (less likely) Fe-As alloy formation and (ii) modification of the phonon density of states, $g(E)$, due to the finite size of the Fe nanoclusters. In fact, a shift of $g(E)$ to lower phonon energies has been observed experimentally in nanocrystalline Fe (Ref. 90) and has been pre- dicted theoretically for metallic nanoclusters, ${ }^{91-93}$ which leads to a reduction of $f$ and $\Theta_{D}$. Further, $g(E)$ at the interface between the continuous Fe film and a semiconductor similar to $\operatorname{GaAs}(001)$, namely, $\operatorname{InAs}(001)$, has been measured by nuclear resonant inelastic $\mathrm{x}$-ray scattering and found to be drastically enhanced at low phonon energies ${ }^{94}$ as compared to that of bulk bcc-Fe, resulting in strongly reduced values of $f$ and $\Theta_{D}$. The strong phonon softening in the Fe nanoclusters observed here and in other systems ${ }^{95}$ will have implications for phonon-related and phonon-assisted phenomena in this system (such as thermodynamical properties, preexponential factors, e.g., in surface diffusion, and $1 / f$ electronic noise).

\section{CONCLUSIONS}

The growth, structure, hyperfine magnetic properties, and lattice vibrational dynamics of uncoated bcc- ${ }^{57} \mathrm{Fe}(001)$ ultrathin films deposited at $\sim 50{ }^{\circ} \mathrm{C}$ onto $\mathrm{GaAs}(001)-(4 \times 6)$ surfaces have been investigated in situ in UHV by STM, LEED/ RHEED, XPS, and ${ }^{57} \mathrm{Fe}$ CEMS. Up to 3.6 ML Fe coverage, RHEED images still display reflections from the GaAs(001) substrate, demonstrating incomplete coalescence of $\mathrm{Fe}$ islands (nanoclusters). This is corroborated in real space by STM, where for $t_{\mathrm{Fe}}=1 \mathrm{ML}$ coverage, small Fe clusters with heights of 1-2 ML are arranged in rows along the [110] direction. Upon increase of the Fe coverage to $2.5 \mathrm{ML}$, the Fe cluster size-distribution becomes more homogeneous, and the clusters appear to be mostly 2 ML high and are connected along the $[-110]$, but not along the [110] direction, suggesting that the onset of ferromagnetism at RT would not arise at $t_{\mathrm{Fe}}=2.5 \mathrm{ML}$. Upon increasing the Fe coverage to $t_{\mathrm{Fe}}=3 \mathrm{ML}$, the surface morphology consists of percolated Fe clusters without preferential orientation, and deposition of $4 \mathrm{ML} F$ leads to a fairly smooth surface with the same surface roughness of $2 \mathrm{ML}$ in height. The effect of intermixing between the Fe overlayers and repelled $\mathrm{Ga}$ and As atoms from the substrate was investigated by XPS. The deposition of $1 \mathrm{ML}$ Fe leads to a moderate increase of the Ga and As $3 d$ binding energies, indicating a substantial change in the $\mathrm{Ga}$ and As coordination and, predominantly, $\mathrm{Fe}-\mathrm{GaAs}$ interactions as the origin of the moderate shift in the binding energy. At $t_{\mathrm{Fe}}$ $=2.5 \mathrm{ML}$, only slight shifts of the binding energy of Ga $3 d$ and As $3 d$ levels are observed, which is attributed to an increase of the Fe-Fe coordination. At larger coverages (4 ML $\mathrm{Fe}$ ), segregation of $\mathrm{Ga}$ atoms appears to occur since an additional XPS satellite is observed below a binding energy of $18 \mathrm{eV}$. This shoulder is attributed to repelled Ga atoms diffusing to the surface of the Fe layer.

Epitaxial bcc- ${ }^{57} \mathrm{Fe}$ ultrathin films at $\mathrm{Fe}$ coverages of 1.9, 2.2 , and $2.5 \mathrm{ML}$ on $\mathrm{GaAs}(001)-(4 \times 6)$ surfaces were investigated in situ in UHV by ${ }^{57} \mathrm{Fe}$ CEMS in zero-external field in the temperature range between $27 \mathrm{~K}$ and room temperature. At such low coverages, these uncoated (free) Fe films consist predominantly of isolated Fe nanoclusters below their percolation threshold. At the lowest temperatures $(27-40 \mathrm{~K})$, we find magnetic ordering for all samples, as evidenced by the appearance of a large mean hyperfine magnetic field $\left\langle B_{h f}\right\rangle$ of $\sim 22-23 \mathrm{~T}$. This value is about the same 
for all Fe coverages investigated, demonstrating that the hyperfine field near a magnetic saturation (extrapolated to $T$ $=0 \mathrm{~K})$ is nearly independent of the Fe cluster size and is an intrinsic property of the magnetically ordered clusters. The observed approximately quasilinear decrease of $\left\langle B_{h f}\right\rangle$ with $T$ is typical for superparamagnetic systems and provides superparamagnetic blocking temperatures $T_{B}$ of $62 \pm 5,80 \pm 10$, and $165 \pm 5 \mathrm{~K}$ for $t_{\mathrm{Fe}}=1.9,2.2$, and $2.5 \mathrm{ML}$, respectively. Our present results demonstrate that the nature of the percolation transition for free (uncoated) Fe nanoclusters on $\mathrm{GaAs}(001)$ in UHV is from superparamagnetism to ferromagnetism and not from paramagnetism to ferromagnetism. The magnetic anisotropy energies $C V$ for the superparamagnetic relaxation are estimated to be 37,48 , and $98 \mathrm{meV}$ for $1.9,2.2$, and 2.5 $\mathrm{ML} \mathrm{Fe}$, respectively. Combined with the average Fe nanoparticle volume, determined by STM, this leads to a large magnetic anisotropy constant $C$ of $\sim 5 \times 10^{5} \mathrm{~J} / \mathrm{m}^{3}$ for $1.9-2.2$ $\mathrm{ML} \mathrm{Fe}$ and of $\sim 8 \times 10^{5} \mathrm{~J} / \mathrm{m}^{3}$ at $2.5 \mathrm{ML} \mathrm{Fe}$. Our values are of the same order of magnitude as the out-of-plane magnetic anisotropy components obtained by in situ FMR on uncoated $\mathrm{Fe} / \mathrm{GaAs}(001)$ at $40 \mathrm{~K}$ and $5 \mathrm{ML}$ coverage ${ }^{53}$ and by in situ Brillouin light scattering on uncoated $\mathrm{Fe} / \mathrm{GaAs}(001)$ at RT. ${ }^{47}$ However, our findings are at variance with those of other groups, ${ }^{34,48}$ who have investigated metal-coated ultrathin Fe films on $\operatorname{GaAs}(001)$ at low temperatures and who do not observe magnetic ordering at coverages $t_{\mathrm{Fe}} \leqslant 2.5 \mathrm{ML}$. This discrepancy emphasizes the importance of in situ experiments in UHV on uncoated $\mathrm{Fe}$ clusters in order to probe their intrinsic magnetic properties. From the $T$ dependence of the Mössbauer spectral area, a strikingly low Debye temperature $\Theta_{D}$ of $(196 \pm 4) \mathrm{K}$ is deduced for uncoated Fe nanoclusters on $\operatorname{GaAs}(001)$ at 1.9-2.2 ML coverage in UHV, indicating strong phonon softening and severe modifications of the phonon density of states in the clusters as compared to bulk bcc-Fe. This has a strong impact on the thermodynamic properties of these clusters.

\section{ACKNOWLEDGMENTS}

We are very grateful to Ulrich von Hörsten for his expert technical assistance and Marco Walterfang for helpful discussions. This work was financially supported by Deutsche Forschungsgemeinschaft (GK 277 and SFB 491), National Science Foundation under Grant No. 044849, and ACSPetroleum Research Foundation (ACS-PRF Award No. 42701-G5).
*Author to whom correspondence should be addressed. roldan@physics.ucf.edu

${ }^{\dagger}$ Permanent address: Tohoku University, Sendai, Japan.

${ }^{1}$ G. Wastlbauer and J. A. C. Bland, Adv. Phys. 54, 137 (2005), and references therein before the year 2005.

${ }^{2}$ G. A. Prinz, Science 250, 1092 (1990).

${ }^{3}$ S. A. Wolf, D. D. Awschalom, R. A. Buhrman, J. M. Daughton, and S. von Molnar, Science 294, 1488 (2001).

${ }^{4}$ I. Zutic, J. Fabian, and S. Das Sarma, Rev. Mod. Phys. 76, 323 (2004).

${ }^{5}$ B. T. Jonker, A. T. Hanbicki, Y. D. Park, G. Itskos, M. Furis, G. Kioseoglåu, A. Petrou, and X. Wei, Appl. Phys. Lett. 79, 3098 (2001).

${ }^{6}$ H. J. Zhu, M. Ramsteiner, H. Kostial, M. Wassermeier, H.-P. Schönherr, and K. H. Ploog, Phys. Rev. Lett. 87, 016601 (2001).

${ }^{7}$ A. T. Hanbicki, O. M. J. van’t Erve, R. Magno, G. Kioseoglåu, C. H. Li, B. T. Jonker, G. Itskos, R. Mallory, M. Yasar, and A. Petrou, Appl. Phys. Lett. 82, 4092 (2003).

${ }^{8}$ O. M. J. van’t Erve, G. Kioseoglåu, A. T. Hanbicki, C. H. Li, B. T. Jonker, R. Mallory, M. Yasar, and A. Petrou, Appl. Phys. Lett. 84, 4334 (2004).

${ }^{9}$ N. C. Gerhardt, S. Hövel, C. Brenner, M. R. Hofmann, F.-Y. Lo, D. Reuter, A. D. Wieck, E. Schuster, W. Keune, and K. Westerholt, Appl. Phys. Lett. 87, 032502 (2005).

${ }^{10}$ C. Adelmann, X. Lou, J. Strand, C. J. Palmstrom, and P. A. Crowell, Phys. Rev. B 71, 121301(R) (2005).

${ }^{11}$ A. T. Hanbicki, B. T. Jonker, G. Itskos, G. Kioseoglou, and A. Petrou, Appl. Phys. Lett. 80, 1240 (2002).

${ }^{12}$ A. Hirohata, Y. B. Xu, C. M. Guertler, J. A. C. Bland, and S. N. Holmes, Phys. Rev. B 63, 104425 (2001).

${ }^{13}$ J. R. Waldrop and R. W. Grant, Appl. Phys. Lett. 34, 630 (1979).
${ }^{14}$ G. A. Prinz and J. J. Krebs, Appl. Phys. Lett. 39, 397 (1981).

${ }^{15}$ J. J. Krebs, B. T. Jonker, and G. A. Prinz, J. Appl. Phys. 61, 2596 (1987).

${ }^{16}$ J. M. Florczak and E. D. Dahlberg, Phys. Rev. B 44, 9338 (1991).

${ }^{17}$ C. Daboo, R. J. Hicken, E. Gu, M. Gester, S. J. Gray, D. E. P. Eley, E. Ahmad, J. A. C. Bland, R. Ploessl, and J. N. Chapman, Phys. Rev. B 51, 15964 (1995).

${ }^{18}$ M. Gester, C. Daboo, R. J. Hicken, S. J. Gray, A. Ercole, and J. A. C. Bland, J. Appl. Phys. 80, 347 (1996).

${ }^{19}$ P. M. Thibado, E. Kneedler, B. T. Jonker, B. R. Bennett, B. V. Shanabrook, and L. J. Whitman, Phys. Rev. B 53, R10481 (1996).

${ }^{20}$ E. Kneedler, P. M. Thibado, B. T. Jonker, B. R. Bennett, B. V. Shanabrook, R. J. Wagner, and L. J. Whitman, J. Vac. Sci. Technol. B 14, 3193 (1996).

${ }^{21}$ E. M. Kneedler, B. T. Jonker, P. M. Thibado, R. J. Wagner, B. V. Shanabrook, and L. J. Whitman, Phys. Rev. B 56, 8163 (1997).

${ }^{22}$ E. M. Kneedler and B. T. Jonker, J. Appl. Phys. 81, 4463 (1997).

${ }^{23}$ Y. B. Xu, E. T. M. Kernohan, D. J. Freeland, A. Ercole, M. Tselepi, and J. A. C. Bland, Phys. Rev. B 58, 890 (1998).

${ }^{24}$ Y. B. Xu, E. T. M. Kernohan, D. J. Freeland, M. Tselepi, A. Ercole, and J. A. C. Bland, J. Magn. Magn. Mater. 198-199, 703 (1999).

${ }^{25}$ D. J. Freeland, Y. B. Xu, E. T. M. Kernohan, M. Tselepi, and J. A. C. Bland, Thin Solid Films 343-344, 210 (1999).

${ }^{26}$ M. Zölfl, M. Brockmann, M. Köhler, S. Kreuzer, T. Schweinböck, S. Miethaner, F. Bensch, and G. Bayreuther, J. Magn. Magn. Mater. 175, 16 (1997).

${ }^{27}$ M. Brockmann, M. Zölfl, S. Miethaner, and G. Bayreuther, J. Magn. Magn. Mater. 198-199, 384 (1999).

${ }^{28}$ A. Filipe, A. Schuhl, and P. Galtier, Appl. Phys. Lett. 70, 129 (1997). 
${ }^{29}$ A. Filipe and A. Schuhl, J. Appl. Phys. 81, 4359 (1997).

${ }^{30}$ B. Lépine, S. Ababou, A. Guivarc'h, G. Jezequel, S. Deputier, R. Guerin, A. Filipe, A. Schuhl, F. Abel, C. Cohen, A. Rocher, and J. J. Crestou, J. Appl. Phys. 83, 3077 (1998).

${ }^{31}$ R. A. Gordon, E. D. Crozier, D.-T. Jiang, T. L. Monchesky, and B. Heinrich, Phys. Rev. B 62, 2151 (2000).

${ }^{32}$ F. Bensch, G. Garreau, R. Moosbühler, G. Bayreuther, and E. Beaurepaire, J. Appl. Phys. 89, 7133 (2001).

${ }^{33}$ R. Moosbühler, F. Bensch, M. Dumm, and G. Bayreuther, J. Appl. Phys. 91, 8757 (2002).

${ }^{34}$ F. Bensch, R. Moosbühler, and G. Bayreuther, J. Appl. Phys. 91, 8754 (2002).

${ }^{35}$ M. Doi, B. Roldan Cuenya, W. Keune, T. Schmitte, A. Nefedov, H. Zabel, D. Spoddig, R. Meckenstock, and J. Pelzl, J. Magn. Magn. Mater. 240, 407 (2002).

${ }^{36}$ J. W. Freeland, I. Coulthard, W. J. Antel, Jr., and A. P. J. Stampfl, Phys. Rev. B 63, 193301 (2001).

${ }^{37}$ Y. Chye, V. Huard, M. E. White, and P. M. Petroff, Appl. Phys. Lett. 80, 449 (2002).

${ }^{38}$ S. J. Steinmüller, M. Tselepi, V. Strom, and J. A. C. Bland, J. Appl. Phys. 91, 8679 (2002).

${ }^{39}$ H. P. Schönherr, R. Nötzel, W. Ma, and K. H. Ploog, J. Appl. Phys. 89, 169 (2001).

${ }^{40}$ K. H. Ploog, J. Herfort, H.-P. Schönherr, M. Moreno, and S. Dhar, J. Cryst. Growth 251, 292 (2003).

${ }^{41}$ B. Roldan Cuenya, M. Doi, W. Keune, S. Hoch, D. Reuter, A. Wieck, T. Schmitte, and H. Zabel, Appl. Phys. Lett. 82, 1072 (2003).

${ }^{42}$ S. McPhail, C. M. Gürtler, F. Montaigne, Y. B. Xu, M. Tselepi, and J. A. C. Bland, Phys. Rev. B 67, 024409 (2003).

${ }^{43}$ O. Thomas, Q. Shen, P. Schieffer, N. Tournerie, and B. Lepine, Phys. Rev. Lett. 90, 017205 (2003).

${ }^{44}$ Y. Zhai, L. Shi, W. Zhang, Y. X. Xu, M. Lu, H. R. Zhai, W. X. Tang, X. F. Jin, Y. B. Xu, and J. A. C. Bland, J. Appl. Phys. 93, 7622 (2003).

${ }^{45}$ S. J. Steinmüller, M. Tselepi, G. Wastlbauer, V. Strom, D. M. Gillingham, A. Ionescu, and J. A. C. Bland, Phys. Rev. B 70, 024420 (2004).

${ }^{46}$ J. S. Claydon, Y. B. Xu, M. Tselepi, J. A. C. Bland, and G. van der Laan, Phys. Rev. Lett. 93, 037206 (2004).

${ }^{47}$ M. Madami, S. Tacchi, G. Carlotti, G. Gubbiotti, and R. L. Stamps, Phys. Rev. B 69, 144408 (2004).

${ }^{48}$ J. Herfort, W. Braun, A. Trampert, H.-P. Schönherr, and K. H. Ploog, Appl. Surf. Sci. 237, 181 (2004).

${ }^{49}$ A. Ionescu, M. Tselepi, D. M. Gillingham, G. Wastlauer, S. J. Steinmüller, H. E. Beere, D. A. Ritchie, and J. A. C. Bland, Phys. Rev. B 72, 125404 (2005).

${ }^{50}$ N. A. Morley, S. L. Tang, M. R. J. Gibbs, E. Ahmad, I. G. Will, and Y. B. Xu, J. Appl. Phys. 97, 10H501 (2005).

${ }^{51}$ L. Giovanelli, G. Panaccione, G. Rossi, M. Fabrizioli, C.-S. Tian, P. L. Gastelois, J. Fujii, and C. H. Back, Phys. Rev. B 72, 045221 (2005).

${ }^{52}$ E. Schuster, W. Keune, F.-Y. Lo, D. Reuter, A. Wieck, and K. Westerholt, Superlattices Microstruct. 37, 313 (2005).

${ }^{53}$ Kh. Zakeri, Th. Kebe, J. Lindner, and M. Farle, Phys. Rev. B 73, 052405 (2006).

${ }^{54}$ P. Schieffer, A. Guivarc'h, C. Lallaizon, B. Lépine, D. Sébilleau, P. Turban, and G. Jézéquel, Appl. Phys. Lett. 89, 161923 (2006).

${ }^{55}$ R. A. Gordon and E. D. Crozier, Phys. Rev. B 74, 165405 (2006).
${ }^{56}$ S. A. Chambers, F. Xu, H. W. Chen, I. M. Vitomirov, S. B. Anderson, and J. H. Weaver, Phys. Rev. B 34, 6605 (1986).

${ }^{57}$ M. W. Ruckman, J. J. Joyce, and J. H. Weaver, Phys. Rev. B 33, 7029 (1986).

${ }^{58}$ T. L. Monchesky, R. Urban, B. Heinrich, M. Klaua, and J. Kirschner, J. Appl. Phys. 87, 5167 (2000).

${ }^{59}$ T. L. Monchesky, A. Enders, R. Urban, K. Myrtle, B. Heinrich, X.-G. Zhang, W. H. Butler, and J. Kirschner, Phys. Rev. B 71, 214440 (2005).

${ }^{60}$ A. Schatz and W. Keune, Surf. Sci. 440, L841 (1999).

${ }^{61}$ R. A. Brand, Nucl. Instrum. Methods Phys. Res. B 28, 417 (1987).

${ }^{62}$ Q.-K. Xue, T. Hashizume, and T. Sakurai, Surf. Sci. 141, 244 (1999).

${ }^{63}$ J. Behrend, M. Wassermeier, L. Däweritz, and K. H. Ploog, Surf. Sci. 342, 63 (1995).

${ }^{64}$ D. K. Biegelsen, R. D. Bringans, J. E. Northrup, and L. E. Swartz, Phys. Rev. B 41, 5701 (1990).

${ }^{65}$ J. F. Moulder, W. F. Stickle, P. E. Sobol, and K. D. Bomben, in Handbook of X-Ray Photoelectron Spectroscopy, edited by J. Chastain and R. C. King, Jr. (Physical Electronics, Eden Prairie, MN, 1995).

${ }^{66}$ L. Zhang, R. Persaud, and T. E. Madey, Phys. Rev. B 56, 10549 (1997).

${ }^{67}$ S. Mirbt, S. Sanyal, C. Isheden, and B. Johansson, Phys. Rev. B 67, 155421 (2003).

${ }^{68}$ S. C. Erwin, S.-H. Lee, and M. Scheffler, Phys. Rev. B 65, 205422 (2002).

${ }^{69}$ D. L. Williamson, in Mössbauer Isomer Shifts, edited by G. K. Shenoy and F. E. Wagner (North-Holland, Amsterdam, 1978), p. 317.

${ }^{70}$ L. R. Newkirk and C. C. Tsuei, Phys. Rev. B 4, 4046 (1971).

${ }^{71}$ G. K. Wertheim, Mössbauer-Effect: Principles and Applications (Academic, New York, 1964), p. 69.

${ }^{72}$ H. Spies, Diploma thesis, Gerhard-Mercator University, Duisburg, Germany, 2000 (in German).

${ }^{73}$ J. L. Dormann, D. Fiorani, and E. Tronc, Adv. Chem. Phys. 98, 283 (1997)

${ }^{74}$ It is not clear whether the oscillations in $P\left(B_{h f}\right)$ observed, in particular, at 40 and $50 \mathrm{~K}$ are physically significant or are an artifact of the mathematical fitting procedure; we think that the overall width of $P\left(B_{h f}\right)$ and the average hf field are not significantly affected by such details of the distribution.

${ }^{75}$ M. F. Thomas and C. E. Johnson, in Mössbauer Spectroscopy, edited by D. P. E. Dickson and F. J. Berry (Cambridge University Press, Cambridge, England, 1986), p. 143. See also Fig. 4.29 in this reference.

${ }^{76}$ F. C. Voogt, T. T. M. Palstra, L. Niesen, O. C. Rogojanu, M. A. James, and T. Hibma, Phys. Rev. B 57, R8107 (1998).

${ }^{77}$ C. L. Chien, J. Appl. Phys. 69, 5267 (1991).

${ }^{78}$ G. Xiao, S. H. Liou, A. Levy, J. N. Taylor, and C. L. Chien, Phys. Rev. B 34, 7573 (1986).

${ }^{79}$ R. S. Preston, S. S. Hanna, and J. Heberle, Phys. Rev. 128, 2207 (1962).

${ }^{80} \mathrm{~S}$. Mørup, J. A. Dumesic, and H. Topsøe, in Applications of Mössbauer Spectroscopy, edited by R. L. Cohen (Academic, New York, 1980), Vol. II.

${ }^{81}$ M. Carbucicchio, M. Rateo, G. Ruggiero, and G. Turilli, Hyperfine Interact. 113, 303 (1998).

${ }^{82}$ E. Fonda and A. Traverse, J. Magn. Magn. Mater. 268, 292 
(2004).

${ }^{83}$ M. M. J. Bischoff, T. Yamada, A. J. Quinn, R. G. P. van der Kraan, and H. van Kempen, Phys. Rev. Lett. 87, 246102 (2001).

${ }^{84}$ M. M. J. Bischoff, T. K. Yamada, and H. van Kempen, Phys. Rev. B 67, 165403 (2003).

${ }^{85}$ M. Košuth, V. Popescu, H. Ebert, and G. Bayreuther, Europhys. Lett. 72, 816 (2005).

${ }^{86}$ Kh. Zakeri, Th. Kebe, J. Lindner, and M. Farle, J. Magn. Magn. Mater. 316, e334 (2007).

${ }^{87}$ G. K. Shenoy, F. E. Wagner, and G. M. Kalvius, in Mössbauer Isomer Shifts, edited by G. K. Shenoy and F. E. Wagner (NorthHolland, Amsterdam, 1978), p. 49.

${ }^{88}$ D. A. Shirley, M. Kaplan, and P. Axel, Phys. Rev. 123, 816 (1961).
${ }^{89}$ R. Lübbers, H. F. Grünsteudel, A. I. Chumakov, and G. Wortmann, Science 287, 1250 (2000).

${ }^{90}$ B. Fultz, C. C. Ahn, E. E. Alp, W. Sturhahn, and T. S. Toellner, Phys. Rev. Lett. 79, 937 (1997).

${ }^{91}$ A. Kara and T. S. Rahman, Phys. Rev. Lett. 81, 1453 (1998).

${ }^{92}$ D. Y. Sun, X. G. Gong, and X. Q. Wang, Phys. Rev. B 63, 193412 (2001).

${ }^{93}$ R. Meyer, L. J. Lewis, S. Prakash, and P. Entel, Phys. Rev. B 68, 104303 (2003).

${ }^{94}$ R. Peters, E. Schuster, B. Roldan Cuenya, W. Keune, W. Sturhahn, T. S. Toellner, and E. E. Alp (unpublished).

${ }^{95}$ B. Roldan Cuenya, A. Naitabdi, J. Croy, W. Sturhahn, J. Y. Zhao, E. E. Alp, R. Meyer, D. Sudfeld, E. Schuster, and W. Keune (unpublished). 